\title{
X-ray emission of $z>2.5$ active galactic nuclei can be obscured by their host galaxies
}

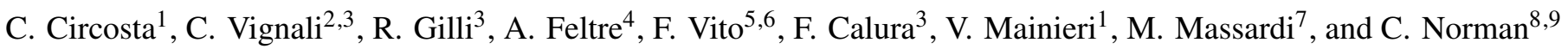

${ }^{1}$ European Southern Observatory, Karl-Schwarzschild-Str. 2, 85748 Garching bei München, Germany e-mail: ccircost@eso.org

2 Dipartimento di Fisica e Astronomia dell'Università degli Studi di Bologna, Via P. Gobetti 93/2, 40129 Bologna, Italy

3 INAF/OAS, Osservatorio di Astrofisica e Scienza dello Spazio di Bologna, Via P. Gobetti 93/3, 40129 Bologna, Italy

${ }^{4}$ Univ. Lyon, Univ. Lyon1, Ens de Lyon, CNRS, Centre de Recherche Astrophysique de Lyon UMR5574, 69230 Saint-Genis-Laval, France

5 Instituto de Astrofisica and Centro de Astroingenieria, Facultad de Fisica, Pontificia Universidad Catolica de Chile, Casilla 306, Santiago 22, Chile

6 Chinese Academy of Sciences South America Center for Astronomy, National Astronomical Observatories, CAS, Beijing 100012, PR China

7 INAF, Istituto di Radioastronomia - Italian ARC, Via Piero Gobetti 101, 40129 Bologna, Italy

8 Department of Physics and Astronomy, Johns Hopkins University, Baltimore, MD 21218, USA

9 Space Telescope Science Institute, 3700 San Martin Drive, Baltimore, MD 21218, USA

Received 14 October 2018 / Accepted 16 January 2019

\begin{abstract}
We present a multiwavelength study of seven active galactic nuclei (AGN) at spectroscopic redshift $>2.5$ in the 7 Ms Chandra Deep Field South that were selected for their good far-infrared (FIR) and submillimeter (submm) detections. Our aim is to investigate the possibility that the obscuration observed in the X-rays can be produced by the interstellar medium (ISM) of the host galaxy. Based on the 7 Ms Chandra spectra, we measured obscuring column densities $N_{\mathrm{H}, \mathrm{X}}$ in excess of $7 \times 10^{22} \mathrm{~cm}^{-2}$ and intrinsic X-ray luminosities $L_{\mathrm{X}}>10^{44} \mathrm{erg} \mathrm{s}^{-1}$ for our targets, as well as equivalent widths for the Fe $\mathrm{K} \alpha$ emission line $\mathrm{EW}_{\text {rest }} \gtrsim 0.5-1 \mathrm{keV}$. We built the UV-toFIR spectral energy distributions (SEDs) by using broadband photometry from the CANDELS and Herschel catalogs. By means of an SED decomposition technique, we derived stellar masses $\left(M_{*} \sim 10^{11} M_{\odot}\right)$, IR luminosities $\left(L_{\mathrm{IR}}>10^{12} L_{\odot}\right)$, star formation rates (SFR $\left.\sim 190-1680 M_{\odot} \mathrm{yr}^{-1}\right)$ and AGN bolometric luminosities $\left(L_{\mathrm{bol}} \sim 10^{46} \mathrm{erg} \mathrm{s}^{-1}\right)$ for our sample. We used an empirically calibrated relation between gas masses and FIR/submm luminosities and derived $M_{\text {gas }} \sim 0.8-5.4 \times 10^{10} M_{\odot}$. High-resolution (0.3-0.7") ALMA data (when available, CANDELS data otherwise) were used to estimate the galaxy size and hence the volume enclosing most of the ISM under simple geometrical assumptions. These measurements were then combined to derive the column density associated with the ISM of the host, which is on the order of $N_{\mathrm{H}, \text { ISM }} \sim 10^{23-24} \mathrm{~cm}^{-2}$. The comparison between the ISM column densities and those measured from the X-ray spectral analysis shows that they are similar. This suggests that at least at high redshift, significant absorption on kiloparsec scales by the dense ISM in the host likely adds to or substitutes that produced by circumnuclear gas on parsec scales (i.e., the torus of unified models). The lack of unobscured AGN among our ISM-rich targets supports this scenario.
\end{abstract}

Key words. galaxies: active - galaxies: evolution - galaxies: star formation - quasars: general - surveys - X-rays: galaxies

\section{Introduction}

The emission observed in active galactic nuclei (AGN) is thought to be produced by gas accretion onto a supermassive black hole (SMBH). Tracing the accretion history of AGN at different cosmic epochs is crucial to understand the way SMBHs have formed and evolved. A key phase of this accretion history occurs at $z=1-3$, when the peak of AGN activity is observed (e.g., Aird et al. 2010; Delvecchio et al. 2014). The amount of gas required to sustain the build-up of the SMBH population in place at high redshift may contribute to the obscuration of the AGN emission itself. Several studies have confirmed that the majority of AGN is obscured by column densities $N_{\mathrm{H}}>10^{22} \mathrm{~cm}^{-2}$ (e.g., Ueda et al. 2014; Buchner et al. 2015), and mounting evidence does support a positive evolution of the obscured AGN fraction with redshift (e.g., Vito et al. 2014, 2018; Aird et al. 2015).

The gas content of galaxies is also observed to have been higher in the past (Carilli \& Walter 2013). This gas fuels star formation activity in galaxies, and the evolution of the star formation rate (SFR) density in the Universe matches that observed for the BH accretion rate (Madau \& Dickinson 2014). The same gas that produces stars is therefore a potential contributor to the obscuration of the AGN. This connection intimately links the history of SMBH accretion to that of the star formation activity of their host galaxies.

Several scaling relations between the $\mathrm{BH}$ mass and the large-scale properties of the host galaxy have been found, such as stellar mass (Magorrian et al. 1998) or velocity dispersion (Ferrarese \& Merritt 2000). The tightness of these relations suggests a direct link between the origin of galaxies and SMBHs, leading to the concept of BH-galaxy coevolution (see Kormendy \& Ho 2013, for a review). This evolutionary scenario matches the $\mathrm{BH}$ accretion phase with strong star formation episodes. Hence, studying the bulk of SMBH accretion also means seeking sites where intense star formation is taking place. The most powerful star-forming sources at high 
redshift are submillimeter galaxies (SMGs), which commonly are detected at a median redshift $z \sim 2-3$ (e.g., Simpson et al. 2014). They are defined as submm sources with flux densities $\gtrsim 1 \mathrm{mJy}$ at $850 \mu \mathrm{m}$, corresponding to typical $L_{\mathrm{IR}} \sim 10^{12} L_{\odot}$ and estimated SFRs $\sim 100-1000 M_{\odot} \mathrm{yr}^{-1}$ (Blain et al. 2002). SMGs present large reservoirs of cold gas, $\gtrsim 10^{10} M_{\odot}$ (e.g., Coppin et al. 2010; Bothwell et al. 2013; Wang et al. 2013a). A significant fraction ( 20\%; Wang et al. 2013b) of SMGs has been found to host X-ray detected AGN, most of which are obscured with $N_{\mathrm{H}}>10^{23} \mathrm{~cm}^{-2}$.

The SMG phase is thought to be part of a broader evolutionary scenario, where a major merger event between gas-rich galaxies (e.g., Hopkins et al. 2006) or an early phase of fast collapse characterizing massive halos (e.g., Lapi et al. 2014, 2018) trigger starburst activity and $\mathrm{BH}$ accretion, funneling the gas toward the center. After this initial phase, when the $\mathrm{BH}$ is obscured by gas and dust, with column densities reaching even the Compton-thick regime (i.e., $N_{\mathrm{H}} \geq 10^{24} \mathrm{~cm}^{-2}$ ), feedback from the $\mathrm{BH}$ and supernova-driven winds disperse the gas, then revealing the system as a bright powerful quasar that eventually evolves into a passive galaxy. The physical properties characterizing the different phases of this evolutionary cycle are not well understood. However, the study of the obscured and active phase, especially for high-redshift sources where most of the mass accretion occurred, can be crucial for better comprehending the interplay between the $\mathrm{BH}$ and its host.

Obscuration of the AGN emission is usually ascribed to a parsec-scale absorber, that is, the nuclear $(\sim 10 \mathrm{pc})$ torus of dust and gas surrounding the central engine, postulated by the unified model (e.g., Urry \& Padovani 1995). However, with the framework of the BH-galaxy coevolution in mind, gas on galaxy-wide scales could also have a role in obscuring the AGN. This role may be not negligible especially at high redshift, when galaxies were smaller (e.g., van der Wel et al. 2014; Shibuya et al. 2015; Allen et al. 2017) and richer in gas content (e.g., Scoville et al. 2017; Tacconi et al. 2018), and therefore featured a denser interstellar medium (ISM).

The potential contribution of the host galaxy in obscuring the AGN has been investigated by Gilli et al. (2014), who found that the column density associated with the ISM of the host can be on the same order as the column density inferred from the X-ray spectral analysis. Their analysis was performed on a single target, specifically, an SMG hosting a Compton-thick AGN at $z=4.755$. This type of study requires multiwavelength data, from the X-rays to the submm, which can be challenging in the distant Universe. On the one hand, X-ray spectra provide us with a direct measurement of the total hydrogen column density along the line of sight affecting the X-ray emission of AGN through absorption and Compton scattering. On the other hand, estimating the column density associated with the ISM of the host galaxy requires measurements of the gas mass (which is dominated by the molecular phase at high redshift, e.g., Calura et al. 2014) and the size of the galaxy. The former can be inferred, for example, via low-J transitions of $\mathrm{CO}$, a commonly used tracer of cold molecular gas in galaxies. An alternative method requires dust emission measurements and the use of an empirical calibration to convert the monochromatic luminosity at $850 \mu \mathrm{m}$ into a molecular gas mass (Scoville et al. 2016, 2017; Privon et al. 2018). As for the sizes, high-resolution observations of the gas and/or dust emission are necessary (e.g., Hodge et al. 2016; Talia et al. 2018).

Following Gilli et al. (2014), we explore the possibility that the obscuration as seen in the X-rays is produced by the ISM of the host galaxy. This study is performed for the first time on a sample of seven X-ray selected AGN, for which we present a multiwavelength analysis in order to characterize both the host galaxy and the active nucleus. The paper is organized as follows. In Sect. 2 we present the dataset used and the sample selection. In Sect. 3 we describe the X-ray spectral extraction procedure and models used for the spectral analysis. In Sect. 4, the code and parameter setup used for modeling the SEDs of our targets are outlined. The results obtained from our analyses as well as the assumptions we made to estimate the molecular gas mass of each source are presented in Sect. 5. We discuss our findings together with the way ISM sizes and column densities have been derived in Sect. 6. We finally draw our conclusions in Sect. 7.

Throughout the paper, a standard $\Lambda \mathrm{CDM}$ cosmology with $\Omega_{\mathrm{M}}=0.3, \Omega_{\Lambda}=0.7$ and $H_{0}=70 \mathrm{~km} \mathrm{~s}^{-1} \mathrm{Mpc}^{-1}$ is assumed (Planck Collaboration XIII 2016).

\section{Dataset and sample selection}

Deep X-ray surveys are very powerful tools because they offer the possibility of efficiently selecting large samples of AGN in the distant Universe, including low-luminosity AGN (e.g., down to $L_{\mathrm{X},[2-10 \mathrm{keV}]} \sim 10^{43} \mathrm{erg} \mathrm{s}^{-1}$, Luo et al. 2017). Our study focuses on the Chandra Deep Field South (CDF-S; Luo et al. 2017), which provides the deepest X-ray spectral information currently available for distant AGN through its $7 \mathrm{Ms}$ exposure. This field benefits from an extraordinary multiband coverage, allowing us to extend our analysis to a broad range of wavelengths. The Great Observatories Origins Deep Survey South field (GOOD-S; Giavalisco et al. 2004) covers, along with the Cosmic Assembly Near-IR Deep Extragalactic Legacy Survey (CANDELS; Grogin et al. 2011), the central area of the CDF$\mathrm{S}$ and about one-third of the whole field. GOODS-S has been imaged with the major facilities providing a wide combination of multi-epoch data available in several bands (e.g., optical imaging with HST/ACS, optical/near-IR observations with the Subaru Suprime-Cam Intermediate Band Filters, observations in the mid-IR in the Spitzer/IRAC and MIPS bands, as well as farIR in the Herschel/PACS and SPIRE bands). The UV-to-mid-IR (MIR) data used in this study are taken from Hsu et al. (2014), who provide photometric data for all the sources detected in the Extended Chandra Deep Field-South (E-CDF-S; Xue et al. 2016; Lehmer et al. 2005). We complemented these data with far-IR (FIR) data from Herschel/PACS and SPIRE, using the catalogs provided by Magnelli et al. (2013) and Oliver et al. (2012), respectively. We used a positional matching radius of 2 " to associate a FIR counterpart with the sources in the UV-to-MIR catalog, taking into account that we used $24 \mu \mathrm{m}$-priored catalogs that in turn are IRAC-3.6 $\mu \mathrm{m}$ priored. Detections with a signalto-noise ratio $S / N<3$ were converted into $3 \sigma$ upper limits. The photometric data used in this work are corrected for Galactic extinction (Schlegel et al. 1998).

In order to select our sample, we searched for X-ray AGN in the CDF-S that satisfy the following requirements:

1. Redshift higher than 2.5 , to find a compromise between the increasing gas content in the host galaxy with redshift and the sample size.

2. Secure spectroscopic redshift, $z_{\text {spec }}$, as given by Luo et al. (2017) (quality flag "Secure"), in order to avoid the large photometric redshift uncertainties that propagate on different measurements.

3. At least one $>3 \sigma$ detection at $\lambda_{\mathrm{obs}} \geq 100 \mu \mathrm{m}$, in order to constrain the emission produced by cold dust that is heated by star formation activity, and as a result, to derive the intrinsic 
Table 1. AGN sample summary.

\begin{tabular}{lccccc}
\hline \hline $\begin{array}{l}\text { XID } \\
(1)\end{array}$ & $\begin{array}{c}\text { CID } \\
(2)\end{array}$ & $\begin{array}{c}\text { XID } \\
(3)\end{array}$ & $\begin{array}{c}\text { RA } \\
(4)\end{array}$ & $\begin{array}{c}\text { Dec } \\
(5)\end{array}$ & $\begin{array}{c}z_{\text {spec }} \\
(6)\end{array}$ \\
\hline 42 & $326^{a}$ & $34^{b}$ & $03^{\mathrm{h}} 31^{\mathrm{m}} 51.95$ & $-27^{\circ} 53^{\prime} 27^{\prime \prime} \cdot 2$ & 2.940 \\
170 & 14781 & 137 & $03^{\mathrm{h}} 32^{\mathrm{m}} 07^{\mathrm{s}} 99$ & $-27^{\circ} 46^{\prime} 57^{\prime \prime} \cdot 2$ & 2.612 \\
337 & 5479 & 262 & $03^{\mathrm{h}} 32^{\mathrm{m}} 18.85$ & $-27^{\circ} 51^{\prime} 35^{\prime \prime} \cdot 7$ & 3.660 \\
539 & 273 & $403^{c}$ & $03^{\mathrm{h}} 32^{\mathrm{m}} 29.27$ & $-27^{\circ} 56^{\prime} 19^{\prime} \cdot 8$ & 4.755 \\
551 & 6294 & 412 & $03^{\mathrm{h}} 32^{\mathrm{m}} 29.86$ & $-27^{\circ} 51^{\prime} 6^{\prime \prime} 1$ & 3.700 \\
666 & 9834 & 490 & $03^{\mathrm{h}} 32^{\mathrm{m}} 35.72$ & $-27^{\circ} 49^{\prime} 16^{\prime \prime} \cdot 4$ & 2.578 \\
746 & 10578 & 546 & $03^{\mathrm{h}} 32^{\mathrm{m}} 39.68$ & $-27^{\circ} 48^{\prime} 51^{\prime \prime} \cdot 1$ & 3.064 \\
\hline
\end{tabular}

Notes. (1) Source identification number in the $7 \mathrm{Ms}$ CDF-S catalog by Luo et al. (2017); (2) CANDELS identification number; (3) source identification number in the 4 Ms CDF-S catalog by Xue et al. (2011); (4) J2000 right ascension and (5) declination of the optical counterparts of the X-ray source as given by Luo et al. (2017); and (6) spectroscopic redshift as given by Luo et al. (2017). ${ }^{(a)}$ ID from GEMS (Häussler et al. 2007). ${ }^{(b)}$ Also known as ALESS57.1. ${ }^{(c)}$ Also known as ALESS73.1.

luminosity at $850 \mu \mathrm{m}$ and estimate the molecular gas mass (Scoville et al. 2016; Privon et al. 2018).

Out of the 29 targets that match criteria 1 and 2 , we found a total of 7 AGN that also satisfy requirement 3. ID, redshift and coordinates of the final sample are presented in Table 1. XID42 is part of the ALESS sample (Chen et al. 2015a). XID337 was studied by Mainieri et al. (2005), who presented a complete SED analysis. XID551 is the first high- $z$ Compton-thick QSO discovered in the CDF-S by Norman et al. (2002) and was also studied by Comastri et al. (2011) in the $3.3 \mathrm{Ms}$ XMM-Newton survey of the CDF-S. XID539 is the most distant Compton-thick AGN known (Gilli et al. 2011), hosted by a luminous SMG (Coppin et al. 2010; De Breuck et al. 2014; Gilli et al. 2014). XID666 is a Compton-thick QSO hosted by an infrared-luminous galaxy studied by Feruglio et al. (2011) and Del Moro et al. (2016). XID170 and XID746 are known since the 1 Ms observation of the CDF-S (Szokoly et al. 2004). It is relevant to mention that all the targets emerging from the sample selection are obscured, meaning that they are characterized by obscuring column densities $N_{\mathrm{H}} \gtrsim 10^{23} \mathrm{~cm}^{-2}$, according to the value given by the $7 \mathrm{Ms}$ catalog and derived using the hardness ratio $\left(\mathrm{HR}=\frac{H-S}{H+S}\right.$, where $H$ and $S$ are the number of counts in the hard $2-7 \mathrm{keV}$ and soft $0.5-2 \mathrm{keV}$ bands, respectively). This would point to a connection between the presence of dust in the ISM that is heated by star formation, and nuclear absorption that may be produced by the same ISM (see Chen et al. 2015b).

\section{X-ray spectral analysis}

\subsection{Spectral extractions}

We used the data products publicly available in the Chandra Data Archive $^{1}$ for each of the 103 observations of the $7 \mathrm{Ms}$ dataset. For each target, the final spectrum was obtained by combining the spectra extracted from each Chandra pointing. Since individual spectral extractions depend on off-axis angle and rollangle, a source lying at the edges of the field of view can be outside some observations. This was the case for XID42 and XID539, the targets with the largest off-axis angle, for which such observations were excluded from the analysis. The off-axis angles of the sample are listed in Table 2 and range between 1.9' and $9.5^{\prime}$.

\footnotetext{
1 http://cxc.harvard.edu/cda/
}

Table 2. Summary of source parameters from the 7 Ms CDF-S data.

\begin{tabular}{lccc}
\hline \hline $\begin{array}{l}\text { XID } \\
(1)\end{array}$ & $\begin{array}{c}\text { Counts } \\
(2)\end{array}$ & $\begin{array}{c}\text { Off-axis angle } \\
(3)\end{array}$ & $\begin{array}{c}\text { Extraction radius } \\
(4)\end{array}$ \\
\hline 42 & $250 \pm 16$ & 9.51 & 7.0 \\
170 & $1807 \pm 43$ & 4.70 & 3.5 \\
337 & $326 \pm 18$ & 3.84 & 2.6 \\
539 & $74 \pm 9$ & 7.96 & 3.5 \\
551 & $707 \pm 27$ & 2.75 & 2.5 \\
666 & $115 \pm 11$ & 1.88 & 2.3 \\
746 & $2056 \pm 45$ & 2.56 & 2.5 \\
\hline
\end{tabular}

Notes. (1) X-ray source ID (see Table 1); (2) net counts in the full $0.5-7 \mathrm{keV}$ band collected for the whole sample in this work, referred to the $7 \mathrm{Ms}$ dataset (errors are computed assuming a Poisson statistics); (3) off-axis angle in arcmin, that is, the angular separation between the X-ray source and the CDF-S average aimpoint, from the $7 \mathrm{Ms}$ source catalog (Luo et al. 2017); and (4) radius, in arcsec, of the circular area selected for the source spectral extraction.

To extract the spectra, we followed the extraction procedure described in Vito et al. (2013). Images were inspected by means of SAOImage DS9 ${ }^{2}$. The source extraction regions were centered on the target coordinates (see Table 1), while the respective backgrounds were taken from nearby source-free regions in the full $7 \mathrm{Ms}$ exposure image. The choice of the extraction radii was made taking into account the source position in the field of view (because the point spread function, PSF, broadens and distorts as the off-axis angle increases) and the number of counts (because fainter sources require smaller radii in order to reduce the number of background counts, hence increasing the $\mathrm{S} / \mathrm{N})$. We selected circular source regions with radii in the range $2.3-7^{\prime \prime}$, and verified that most of the counts were included. The values chosen for each source are reported in Table 2. Background regions were chosen in nearby areas that are free from contamination due to either close detected objects or the source itself. The background extraction region is larger than the corresponding source extraction region by a factor of $\sim 10-15$ in order to ensure a good sampling of the background itself.

Spectra, response matrices, and ancillary files were extracted with the specextract tool included in the Chandra Interactive Analysis of Observations ${ }^{3}$ (CIAO, v.4.8) software suite. The final spectra were grouped to one count per bin with the grppha tool, and the Cash statistics with direct background subtraction was adopted (Cash 1979; Wachter et al. 1979). The net counts in the full $(0.5-7 \mathrm{keV})$ band for each source are reported in Table 2. They range between $\sim 74$ and $\sim 2056$, with a median value of $\sim 326$, and are in good agreement with the values reported in the Luo et al. (2017) catalog.

Some spectra were analyzed in a narrower energy range (e.g., XID42, XID539) in order to exclude spectral regions that are affected by a high background and to maximize the S/N.

\subsection{Spectral models}

To derive obscuring column densities and X-ray luminosities, we performed a spectral analysis using XSPEC ${ }^{4}$ (Arnaud 1996), v.12.8.2. Because of the low photon statistics, we first adopted a simple power-law model including Galactic

\footnotetext{
2 http://hea-www.harvard.edu/RD/ds9/

3 http://cxc.harvard.edu/ciao/

4 https://heasarc.gsfc.nasa.gov/xanadu/xspec/
} 
absorption $^{5}$ (POWERLAW and PHABs models in XSPEC). The spectral slopes were found to be significantly flatter than the typical intrinsic slope of AGN ( $\Gamma=1.8 \pm 0.2$; e.g., Nandra \& Pounds 1994; Mainieri et al. 2002; Mateos et al. 2005; Tozzi et al. 2006). These hard slopes $(\Gamma \sim 0.0-1.0)$ are in fact characteristic of obscured sources with low-counting statistics (e.g., Del Moro et al. 2016), and if coupled with prominent iron $\mathrm{K} \alpha$ emission features (EW $\gtrsim 1 \mathrm{keV}$ ), they are highly suggestive of heavy obscuration (e.g., Feruglio et al. 2011).

We therefore adopted more complex models to fit the spectra while keeping a minimum number of free parameters. All models have fixed geometric parameters of the obscuring material because these are not known a priori and the data quality does not allow us to obtain them from the fitting process itself. For the majority of the targets, we could not simultaneously place tight constraints on the photon index and column density because these parameters are degenerate for low-count spectra. Hence we fixed the photon index to 1.8. Because the AGN emission is obscured, we also fixed the width of the iron line to $10 \mathrm{eV}$, which only accounts for the narrow component produced by the obscuring medium far away from the central black hole (e.g., Risaliti \& Elvis 2004). We used the models described below.

- A transmission-dominated model, which reproduces the fraction of the primary emission transmitted through the obscuring medium. It is modeled by PLCABs (Yaqoob 1997), which considers transmitted emission for a cold, spherical, and uniform distribution of matter surrounding an X-ray source. This model takes into account Compton scattering and works for column densities up to $\sim 5 \times 10^{24} \mathrm{~cm}^{-2}$, as well as a maximum observed energy of $10-18 \mathrm{keV}$. We added a Gaussian line (zGAuss) to model the iron line and an unabsorbed power law to account for the soft-energy emission component (e.g., radiation that is scattered or leaking from the absorber). The photon index of this secondary power law is the same as that adopted for the primary one, as in the case of Thomson scattering.

- A reflection-dominated model, which implies $N_{\mathrm{H}} \gtrsim 10^{25} \mathrm{~cm}^{-2}$, therefore the direct nuclear emission is entirely absorbed and only emission reflected by the obscuring medium can be observed. It is parametrized by the PEXrAV model (Magdziarz \& Zdziarski 1995), fixing $\Gamma=1.8$, and by a Gaussian line. Moreover, we fixed the cutoff energy to $100 \mathrm{keV}$ and the viewing angle to the default value of $60^{\circ}$.

- The MYTorus model (Murphy \& Yaqoob 2009), which adopts a toroidal geometry for the reprocessor (a tube-like, azimuthally symmetric torus) with a half-opening angle of $60^{\circ}$ and assumes that the reprocessing material is uniform, neutral, and cold. It is valid for column densities in the range $10^{22}-10^{25} \mathrm{~cm}^{-2}$. This model self-consistently reproduces the main components that usually characterize AGN emission (i.e., the "transmitted" and the "reflected" continuum, and the emission line). The inclination of the line of sight is fixed to $75^{\circ}$. We adopted a power-law continuum as primary spectrum, with the photon index fixed to 1.8 and a maximum energy $E_{\mathrm{T}}=500 \mathrm{keV}$ (even if a lower value is not expected to significantly affect our results, given the spectral energy range).

The results of the X-ray spectral analysis are presented in Sect. 5.1. Notes on individual targets are reported in Appendix A.

\footnotetext{
5 The Galactic column density along the line of sight to the CDF-S is $N_{\mathrm{H}}=8.8 \times 10^{19} \mathrm{~cm}^{-2}$ (e.g., Stark et al. 1992).
}

\section{SED-fitting analysis}

We analyzed the multiwavelength data of our targets in order to derive stellar masses, SFRs, and AGN bolometric luminosities, as well as to model the long-wavelength emission and infer the $850 \mu \mathrm{m}$ luminosity (Scoville et al. 2016). To this aim, we used the SED-fitting code originally presented by Fritz et al. (2006) and Hatziminaoglou et al. (2008) and improved by Feltre et al. (2013). For a detailed description of the code, we refer to Feltre et al. (2013) and summarize below some of its main features.

The code adopts a multicomponent fitting approach that accounts for three distinct emission components: (i) stellar emission, which mainly prevails between $\sim 0.3$ and $\sim 5 \mu \mathrm{m}$ (rest frame); (ii) emission due to hot dust heated by the AGN whose emission peaks in the mid-infrared (MIR); (iii) emission by cold dust dominating the FIR regime that is associated with starforming activity.

The first component was modeled with a set of simple stellar populations (SSPs) of solar metallicity and assuming a Salpeter (1955) IMF, convolved with an exponentially declining star formation history (SFH), the so-called direct- $\tau$ model (e.g., Santini et al. 2015), in a time interval ranging between the formation redshift of the galaxy $z_{\text {form }}$ and the source redshift $z$ :

$\operatorname{SFR}(t)=\left(\frac{T_{\mathrm{G}}-t}{T_{\mathrm{G}}}\right) \exp \left(-\frac{T_{\mathrm{G}}-t}{T_{\mathrm{G}} \cdot \tau_{\mathrm{B}}}\right)$,

where $T_{\mathrm{G}}$ is the age of the galaxy (or the age of the oldest SSP), which depends on $z_{\text {form }}$, and $\tau_{\mathrm{B}}$ is the duration of the initial burst normalized to the age of the galaxy. The effect of attenuation was taken into account adopting the Calzetti et al. (2000) law, and applying a common value of attenuation to stars of all ages.

The AGN contribution was modeled with the templates presented by Fritz et al. (2006) and updated by Feltre et al. (2012), which assume that dust, composed of silicate and graphite, is smoothly distributed around the central engine with a flared-disk geometry. They have been extensively used in various analyses at different redshifts (Hatziminaoglou et al. 2010; Vignali et al. 2011; Pozzi et al. 2012). The extent and morphology of the nuclear dust distribution were resolved through high-resolution interferometric observations in the MIR revealing a clumpy or filamentary dust structure (Jaffe et al. 2004; Tristram et al. 2007; Burtscher et al. 2013). However, models assuming both a smooth and clumpy (e.g., Nenkova et al. 2008a,b) dust distribution are widely used and provide a good reproduction of the observed AGN SEDs. According to Feltre et al. (2012), the majority of the differences in the model SEDs are mainly due to different model assumptions and not to the clumpiness or smoothness of the dust distribution. We here focus on the global characteristics of the SEDs and not on the details of the torus structure and geometry.

Finally, the cold dust component was modeled with empirical templates that are representative of starburst galaxies (such as Arp 220, M 82, and NGC 4102; see Polletta et al. 2007). The best fit was determined by a standard $\chi^{2}$ minimization. The results of the SED-fitting analysis are presented in Sect. 5.2. Notes on individual targets are reported in Appendix A.

\section{Results}

\subsection{X-ray spectral analysis}

The best-fit parameters obtained from the X-ray spectral analysis (see Sect. 3.2) are reported in Table 3 for each model we 
Table 3. Best-fit parameters of the X-ray spectral analysis.

\begin{tabular}{|c|c|c|c|c|c|c|c|c|}
\hline $\begin{array}{l}\text { XID } \\
(1)\end{array}$ & $\begin{array}{c}\text { Model } \\
(2)\end{array}$ & $\begin{array}{c}\text { C-stat/d.o.f. } \\
\text { (3) }\end{array}$ & $\begin{array}{l}N_{\mathrm{H}} \\
(4)\end{array}$ & $\begin{array}{c}\Gamma \\
(5)\end{array}$ & $\begin{array}{c}E_{\mathrm{Fe} \text { line }} \\
(6)\end{array}$ & $\begin{array}{l}\text { EW } \\
(7)\end{array}$ & $\begin{array}{c}F_{[2-10 \mathrm{keV}]} \\
(8)\end{array}$ & $\begin{array}{c}L_{[2-10 \mathrm{keV}]} \\
\quad(9)\end{array}$ \\
\hline \multirow[t]{3}{*}{42} & Transmission & $146.9 / 172$ & $2.0_{-0.9}^{+1.0}$ & 1.8 & $6.57_{-0.35}^{+0.20}$ & $386_{-288}^{+335}$ & $3.0_{-0.8}^{+0.8}$ & $1.9_{-0.6}^{+0.8}$ \\
\hline & Reflection & $154.8 / 173$ & - & 1.8 & $6.54_{-0.36}^{+0.19}$ & $504_{-339}^{+504}$ & $6.7_{-0.9}^{+1.0}$ & $34.5_{-5.1}^{+5.0}$ \\
\hline & MYTorus & $151.8 / 177$ & $2.4_{-0.9}^{+1.0}$ & 1.8 & 6.4 & $290_{-261}^{+394}$ & $3.4_{-0.6}^{+0.6}$ & $2.2_{-0.6}^{+1.0}$ \\
\hline \multirow[t]{3}{*}{170} & Transmission & $355.7 / 391$ & $0.7_{-0.1}^{+0.1}$ & 1.8 & 6.4 & $<141$ & $3.7_{-0.2}^{+0.2}$ & $1.7_{-0.2}^{+0.2}$ \\
\hline & Reflection & $972.5 / 389$ & - & 1.8 & 6.4 & $260_{-123}^{+137}$ & $9.3_{-0.4}^{+0.5}$ & $33.9_{-1.7}^{+1.2}$ \\
\hline & MYTorus & $354.3 / 389$ & $0.7_{-0.1}^{+0.1}$ & 1.8 & 6.4 & $<150$ & $3.7_{-0.1}^{+0.4}$ & $1.6_{-0.1}^{+0.1}$ \\
\hline \multirow[t]{3}{*}{337} & Transmission & $207.4 / 240$ & $10.0_{-2.0}^{+3.0}$ & 1.8 & $6.25_{-0.24}^{+0.27}$ & $610_{-508}^{+596}$ & $1.8_{-0.3}^{+0.2}$ & $3.1_{-0.3}^{+1.3}$ \\
\hline & Reflection & $197.4 / 242$ & - & 1.8 & 6.32 & $<503$ & $1.6_{-0.2}^{+0.3}$ & $12.0_{-1.0}^{+2.1}$ \\
\hline & MYTorus & $190.7 / 241$ & $14.0_{-3.4}^{+6.8}$ & 1.8 & 6.4 & $502_{-391}^{+537}$ & $2.0_{-0.4}^{-0.2}$ & $3.8_{-1.2}^{+2.7}$ \\
\hline \multirow[t]{3}{*}{539} & Transmission & $29.2 / 45$ & $17.0_{-6.8}^{+11.7}$ & 1.8 & $6.91_{-0.21}^{+0.39}$ & $3240_{-2946}^{+2325}$ & $1.5_{-1.2}^{+0.3}$ & $6.7_{-4.0}^{+27.0}$ \\
\hline & Reflection & $32.4 / 47$ & - & 1.8 & 6.9 & $<2089$ & $1.0_{-0.2}^{+0.4}$ & $14.5_{-2.7}^{+4.5}$ \\
\hline & MYTorus & $30.9 / 45$ & $15.5_{-6.9}^{+24.9}$ & 1.8 & $6.95_{-0.27}^{+0.59}$ & $2618_{-1973}^{+2514}$ & $1.5_{-1.2}^{+0.4}$ & $5.0_{-1.7}^{+16.8}$ \\
\hline \multirow[t]{3}{*}{551} & Transmission & $268.1 / 307$ & $11.8_{-1.9}^{+2.4}$ & 1.8 & $6.53_{-0.14}^{+0.14}$ & $630_{-310}^{+338}$ & $2.6_{-0.3}^{+0.3}$ & $5.0_{-1.1}^{+1.7}$ \\
\hline & Reflection & $282.1 / 309$ & - & 1.8 & $6.56_{-0.17}^{+0.17}$ & $376_{-230}^{+259}$ & $2.4_{-0.2}^{+0.5}$ & $19.2_{-1.5}^{+1.1}$ \\
\hline & MYTorus & $276.5 / 316$ & $9.5_{-1.7}^{+1.7}$ & 1.8 & 6.4 & $382_{-267}^{+313}$ & $2.6_{-0.3}^{+0.3}$ & $4.1_{-0.7}^{+0.9}$ \\
\hline \multirow[t]{3}{*}{666} & Transmission & $83.6 / 113$ & $32.8_{-8.4}^{+15.4}$ & 1.8 & $6.44_{-0.11}^{+0.09}$ & $1470_{-560}^{+980}$ & $0.9_{-0.2}^{+0.4}$ & $5.6_{-1.2}^{+1.2}$ \\
\hline & Reflection & $87.9 / 114$ & - & 1.8 & $6.44_{-0.10}^{+0.11}$ & $\begin{array}{r}1707_{-712}^{+895} \\
\end{array}$ & $0.6_{-0.2}^{-0.2}$ & $3.0_{-0.4}^{+0.2}$ \\
\hline & MYTorus & $85.9 / 115$ & $>39$ & 1.8 & 6.4 & $1589_{-524}^{+477}$ & $1.3_{-0.2}^{+3.2}$ & $>3.4$ \\
\hline \multirow[t]{3}{*}{746} & Transmission & $372.7 / 382$ & $5.5_{-0.3}^{+0.4}$ & 1.8 & 6.4 & $<100$ & $7.0_{-0.4}^{+0.3}$ & $6.1_{-0.5}^{+0.6}$ \\
\hline & Reflection & $492.8 / 376$ & - & 1.8 & $6.25_{-0.15}^{+0.21}$ & $<325$ & $8.2_{-0.3}^{+0.3}$ & $43.5_{-1.2}^{+1.5}$ \\
\hline & MYTorus & $368.8 / 382$ & $5.5_{-0.3}^{+0.3}$ & 1.8 & 6.4 & $<204$ & $7.2_{-0.4}^{+0.3}$ & $6.4_{-0.5}^{+0.6}$ \\
\hline
\end{tabular}

Notes. (1) X-ray source ID (see Table 1); (2) model used to fit the source spectrum; (3) ratio between the Cash statistic value and the number of degrees of freedom; (4) column density in units of $10^{23} \mathrm{~cm}^{-2}$; (5) spectral index; (6) rest-frame energy of the iron emission line in keV; (7) rest-frame equivalent width of the iron line in $\mathrm{eV}$; (8) observed flux in the hard $(2-10 \mathrm{keV})$ band, in units of $10^{-15} \mathrm{erg} \mathrm{cm}^{-2} \mathrm{~s}^{-1}$; and $(9)$ rest-frame absorption corrected luminosity in the hard $(2-10 \mathrm{keV})$ band, in units of $10^{44} \mathrm{erg} \mathrm{s}^{-1}$. Errors are given at the $90 \%$ confidence level.

used in the fitting procedure. Figure 1 shows X-ray spectra for the whole sample fit by the transmission model. Errors are given at the $90 \%$ confidence level for one parameter of interest (Avni 1976). The whole sample is characterized by very high column densities in the range $\sim 7 \times 10^{22}-3 \times 10^{24} \mathrm{~cm}^{-2}$. In particular, four out of seven sources are Compton-thick candidates. The equatorial column densities obtained with the MYTorus model were converted into the corresponding value along the line of sight (Murphy \& Yaqoob 2009). An iron emission line is detected in five out of seven sources, and in terms of EW, it is consistent within the errors with the prominent line $(\mathrm{EW} \sim 1 \mathrm{keV})$ that is typically observed in obscured AGN spectra. Because of the limited photon statistics, some physical quantities are poorly constrained and characterized by loose limits.

The hard X-ray luminosities were computed in the restframe energy range $2-10 \mathrm{keV}$ and corrected for absorption. These luminosities were found to be in the quasar luminosity domain with values in the range $(2-7) \times 10^{44} \mathrm{erg} \mathrm{s}^{-1}$. Because there is no information on the intrinsic luminosity in the pure reflection model, we estimated it by assuming a reflection efficiency (i.e., the observed-to-intrinsic luminosity ratio) of $2 \%$ in the $2-10 \mathrm{keV}$ band. This efficiency is admittedly very uncertain because it depends on the exact geometry of the absorbing-reflecting medium, but we note that reflection efficiencies on the order of $\sim 1-3 \%$ have been reported in the literature (Maiolino et al. 1998; Comastri et al. 2010; Baloković et al.
2014; Ricci et al. 2017) and are usually assumed for Comptonthick AGN in synthesis models of the X-ray background (Gilli et al. 2007; Akylas et al. 2012). The observed fluxes in the same energy range are between $(1-7) \times 10^{-15} \mathrm{erg} \mathrm{cm}^{-2} \mathrm{~s}^{-1}$.

Errors on luminosities were derived taking into account the uncertainties on the column density as well as on the flux. Specifically, we considered a $90 \%$ confidence level for two parameters of interest (column density and power-law normalization). Our sample partially overlaps those studied by Liu et al. (2017) and Vito et al. (2018). Our results are in good agreement with their analysis.

\subsection{SED decomposition}

In Table 4 we report the most relevant physical parameters derived by fitting the SEDs of our targets: $M_{*}$, the stellar mass of the galaxy; $L_{\mathrm{IR}}$, the total infrared luminosity integrated in the $8-1000 \mu \mathrm{m}$ rest-frame interval; $L_{\mathrm{bol}}$, the AGN bolometric luminosity; $E(B-V)$, the total attenuation to the stellar emission; $f_{\mathrm{AGN}}$, the fractional AGN contribution to the total infrared luminosity in the range $8-1000 \mu \mathrm{m}$; SFR, the star formation rate obtained from $L_{\mathrm{IR}}$ by using the Kennicutt (1998) calibration and subtracting the AGN fraction; and $M_{\text {gas }}$, the gas mass (atomic and molecular hydrogen).

Our galaxies are found to be massive, with stellar masses in the range $(1.7-4.4) \times 10^{11} M_{\odot}$ and characterized by an intense IR 

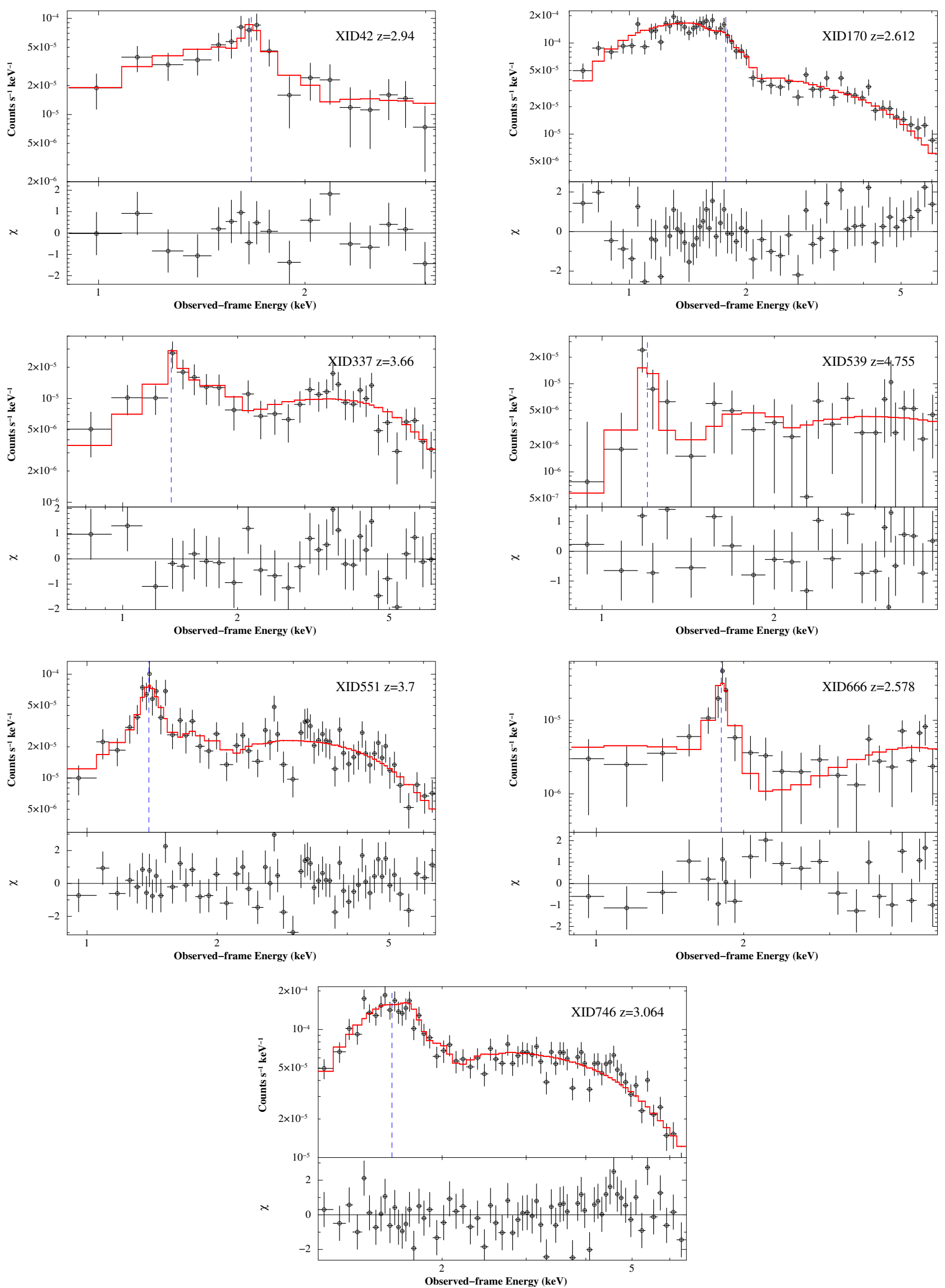

Fig. 1. 7 Ms Chandra spectra of the targets fit using the transmission model. Observations are shown in black and the best-fit model in red. The data-to-model ratios in units of $\sigma$ are shown at the bottom of each panel. The spectra are rebinned here for presentation purposes. The position of the iron line, as reported in Table 3, is marked by a blue dashed line. 
Table 4. Best-fit parameters of the SED decomposition.

\begin{tabular}{lccccccc}
\hline \hline $\begin{array}{l}\mathrm{XID} \\
(1)\end{array}$ & $\begin{array}{c}M_{*} \\
(2)\end{array}$ & $\begin{array}{c}L_{\mathrm{IR}} \\
(3)\end{array}$ & $\begin{array}{c}L_{\text {bol }} \\
(4)\end{array}$ & $\begin{array}{c}E(B-V) \\
(5)\end{array}$ & $\begin{array}{c}f_{\mathrm{AGN}} \\
(6)\end{array}$ & $\begin{array}{c}\mathrm{SFR} \\
(7)\end{array}$ & $\begin{array}{c}M_{\text {gas }} \\
(8)\end{array}$ \\
\hline 42 & $2.16 \pm 0.65$ & $9.62 \pm 1.92$ & $2.60 \pm 0.78$ & 0.31 & 0.01 & $1679 \pm 336$ & $5.41 \pm 3.21$ \\
170 & $1.74 \pm 0.52$ & $1.57 \pm 0.31$ & $1.89 \pm 0.57$ & 0.24 & 0.02 & $276 \pm 55$ & $0.87 \pm 0.51$ \\
337 & $3.43 \pm 1.03$ & $1.01 \pm 0.20$ & $3.11 \pm 0.93$ & 0.24 & 0.09 & $192 \pm 38$ & $0.85 \pm 0.51$ \\
539 & $2.15 \pm 0.64$ & $4.90 \pm 0.98$ & $2.66 \pm 0.80$ & 0.37 & 0.02 & $864 \pm 173$ & $4.55 \pm 2.70$ \\
551 & $2.19 \pm 0.66$ & $2.27 \pm 0.45$ & $2.92 \pm 0.88$ & 0.36 & 0.14 & $456 \pm 91$ & $0.88 \pm 0.52$ \\
666 & $4.41 \pm 1.32$ & $4.90 \pm 0.98$ & $4.82 \pm 1.44$ & 0.38 & 0.03 & $872 \pm 175$ & $2.76 \pm 1.63$ \\
746 & $4.33 \pm 1.30$ & $1.44 \pm 0.29$ & $2.84 \pm 0.85$ & 0.48 & 0.17 & $299 \pm 60$ & $0.80 \pm 0.47$ \\
\hline
\end{tabular}

Notes. (1) X-ray source ID (see Table 1); (2) stellar mass in units of $10^{11} M_{\odot}$; (3) total infrared luminosity integrated in the rest-frame 8-1000 $\mu \mathrm{m}$ range in units of $10^{12} L_{\odot}$; (4) AGN bolometric luminosity in units of $10^{12} L_{\odot}$; (5) attenuation to the stellar emission; (6) fractional AGN contribution to the total IR luminosity; (7) SFR in units of $M_{\odot} \mathrm{yr}^{-1}$; and (8) $M_{\mathrm{gas}}=M_{\mathrm{H}_{2}}+M_{\mathrm{HI}}$ in units of $10^{10} M_{\odot}$. Errors are given at the $68 \%$ confidence level. Relative errors are $\sim 30 \%$ for stellar masses and $\sim 20 \%$ for IR luminosities and AGN bolometric luminosities.

emission, $L_{\mathrm{IR}}=(1.0-9.6) \times 10^{12} L_{\odot}$. In terms of AGN bolometric luminosities, our targets are in the quasar regime, with values between $1.9 \times 10^{12}$ and $4.8 \times 10^{12} L_{\odot}$. The AGN contributes to the IR $8-1000 \mu$ m luminosity up to a few tens of percent. The attenuation to the host galaxy emission is in the range $E(B-V)=$ $0.24-0.48$

We determined the uncertainties on the best-fit parameters by considering all the acceptable solutions within $1 \sigma$ confidence level, which means within a given range $\Delta \chi^{2}$ that depends on the free parameters of the SED-fitting procedure. There are 11 free parameters (see Pozzi et al. 2012): 6 are related to the AGN (see Feltre et al. 2012), 2 to the stellar component $\left(\tau_{\mathrm{B}}\right.$ and $E(B-V)), 1$ to the starburst component (i.e., the selected best-fit template among the starburst library), and 2 further free parameters are represented by the normalizations of the stellar and starburst components. The normalization of the AGN component, instead, is estimated by difference after the other two components (the stellar and FIR components) were fixed by the fitting procedure. Therefore, we considered all the solutions within a $\chi^{2}$ interval $\Delta \chi^{2}=\chi^{2}-\chi_{\min }^{2} \lesssim 12.65$ (Lampton et al. 1976). The resulting relative errors are $\sim 20 \%$ for bolometric as well as IR luminosities, and of a few percent for the stellar masses. This uncertainty is clearly underestimated and is on the order of the statistical errors of the photometric measurements. Comparisons between the stellar masses obtained with the code used in this analysis and other codes (adopting different libraries and IMFs) provide instead a scatter of $\sim 30 \%$. A similar range was also found by Santini et al. (2015), who investigated the influence of systematic effects in the stellar mass estimate produced by different assumptions, mainly due to poor constraints on the stellar population properties (e.g., metallicity, attenuation curves, and IMF) and the lack of a proper reconstruction of the SFH. They collected stellar mass measurements for the sources observed in the CANDELS field (where our targets lie) by ten teams in the CANDELS collaboration, who fit the same photometry, but adopted different assumptions. The comparison of the resulting estimates was quite satisfactory: the majority of the results were around the median value. They therefore claimed that the stellar mass is a stable parameter against the different assumptions, except for the IMF, which introduces a constant offset ${ }^{6}$. They also quantified the scatter around the median value, which is roughly $25 \%-35 \%$. We compared our measurements of the stellar mass with the results presented in their GOODS-S catalog ${ }^{7}$, in particular with the median values and the results obtained with the method whose assumptions are the most similar to ours (method $2 \mathrm{~d}_{\tau}$ in Santini et al. 2015), that is, the $\chi^{2}$ minimization to estimate the goodness of fits, the Salpeter IMF, an exponentially declining SFH, and the Calzetti attenuation law. The quantity $\left\langle\log \left(M_{*}\right.\right.$, literature $\left.\left./ M_{* \text {, this work }}\right)\right\rangle$ is equal to -0.13 dex and -0.07 dex for their median stellar masses (rescaled to a Salpeter IMF) and those obtained with the method $2 \mathrm{~d}_{\tau}$, respectively. The standard deviation is $0.1 \mathrm{dex}$ in both cases. According to the results mentioned above, we assumed a relative error of $30 \%$ for the stellar masses derived in this work.

Overall, the observed SEDs, shown in Fig. 2, are well reproduced by the models. The optical/NIR regime of our SEDs is densely sampled by several photometric datapoints. The AGN contamination to the optical/NIR regime is negligible, as can be seen from the best fits, because we study obscured AGN. The coverage is sparser in the MIR and FIR regimes. The Spitzer/MIPS data at $24 \mu \mathrm{m}$ account for the wavelength range where the AGN emission dominates, but in our targets, the AGN contribution can be important in this regime. The FIR part of the SEDs is differently sampled for the different sources. In general, all the targets have Herschel/PACS and SPIRE photometry, to which we added SCUBA (Rigopoulou et al. 2009) and ALMA submm data (in Bands 7, 6, and/or 4), when available. ALMA data constrain the declining part of the FIR peak (at long wavelengths), which corresponds to the Rayleigh-Jeans tail, associated with dust in the optically thin regime. The dust continuum can therefore be used as an indicator of dust mass, and through the dust-to-gas ratio, of the ISM mass in the galaxy (see Sect. 5.3). The FIR data also allowed us to estimate the SFRs of the sample, which is characterized by an intense starformation activity, with values in the range between $\sim 190$ and $\sim 1680 M_{\odot} \mathrm{yr}^{-1}$.

We compared our estimates of X-ray luminosities with those predicted by the relations found by Lusso et al. (2012) with bolometric luminosities, and Gandhi et al. (2009) with $12.3 \mu \mathrm{m}$ luminosities. Our results are in good agreement with the predicted values, and they agree on average to within a difference of 0.1 dex and 0.3 dex for the Lusso et al. (2012) and Gandhi et al. (2009) relations, respectively.

\footnotetext{
6 In order to rescale the stellar mass from the Salpeter to the Chabrier IMF, 0.24 dex needs to be subtracted.
}

7 http://candels.ucolick.org/data_access/GOODS-S.html 

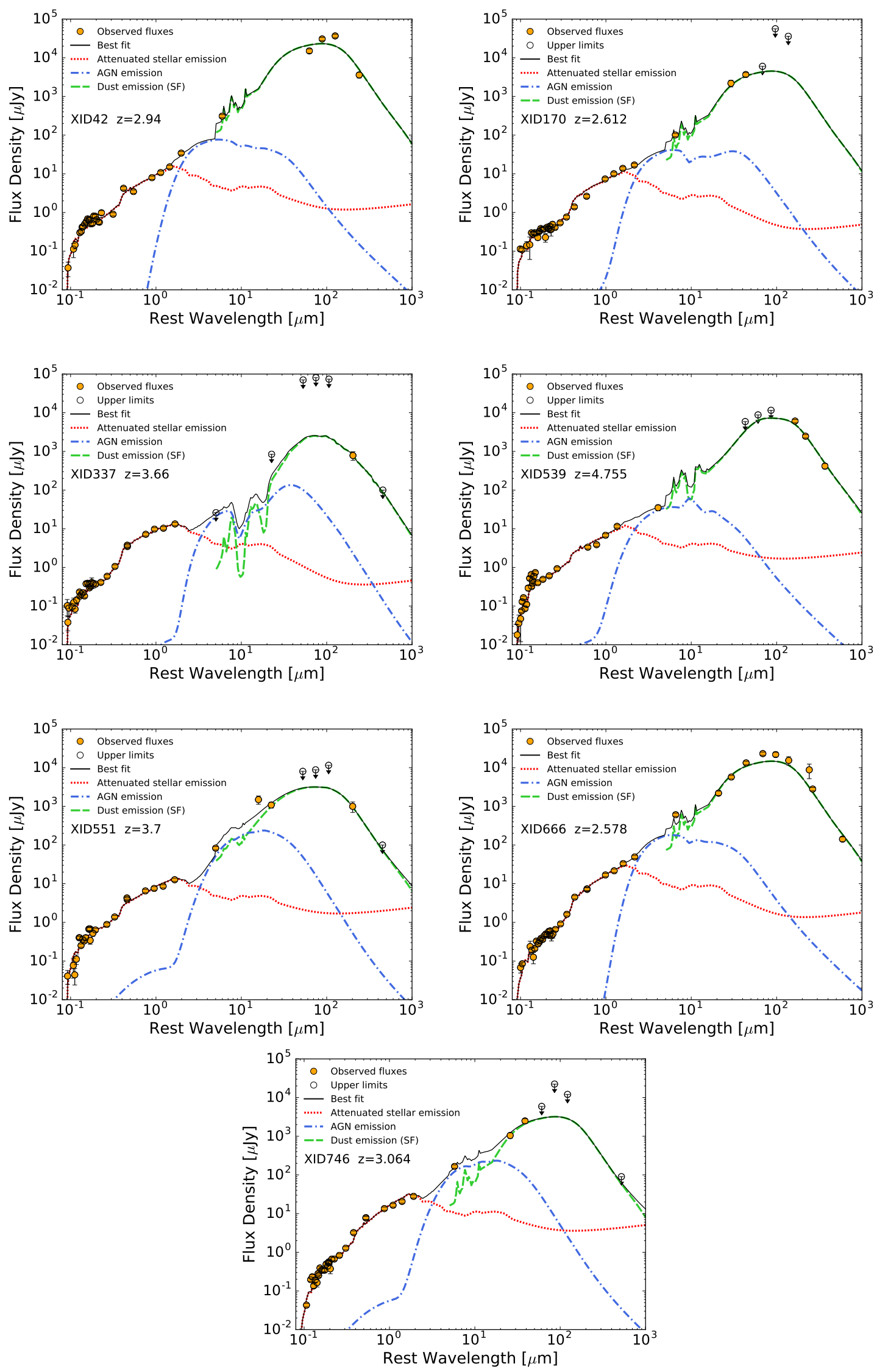

Fig. 2. Spectral decomposition of the rest-frame SEDs of the target sample. The X-ray source ID (from Luo et al. 2017) and the redshift are shown in the middle-left part of each panel. The orange filled dots depict photometric data, and empty dots indicate $3 \sigma$ upper limits. The black solid line is the total best-fit model, the red dotted line represents the stellar emission attenuated by dust, the AGN model is reproduced by the blue dot-dashed line, and the green dashed line accounts for dust emission heated by star formation. 


\subsection{Gas content of the host galaxies}

In order to estimate the gas content of the host galaxies, we derived the molecular gas mass, which is the dominant component in these sources, using the results obtained by Scoville et al. (2016). They analyzed both long-wavelength dustcontinuum emission and $\mathrm{CO}(1-0)$ line luminosities for a large sample of galaxies that consists of local star-forming galaxies, low-z ultra-luminous infrared galaxies (ULIRGs) and high- $z$ SMGs. All galaxies show the same linear correlation between $\mathrm{CO}(1-0)$ luminosity $L_{\mathrm{CO}}^{\prime}$ and the luminosity at $850 \mu \mathrm{m} L_{850 \mu \mathrm{m}}$ rest frame, $L_{\mathrm{CO}}^{\prime}=3.02 \times 10^{-21} L_{850 \mu \mathrm{m}}$ (see left panel of Fig. 1 in Scoville et al. 2016), probing the molecular gas mass and the dust emission, respectively. We estimated the luminosity at $850 \mu \mathrm{m}$ rest frame from the model SEDs of our targets and recovered $L_{\mathrm{CO}}^{\prime}$ through the observed correlation. The molecular gas mass can then be quantified assuming a CO-to- $\mathrm{H}_{2}$ conversion factor $\alpha_{\mathrm{CO}}, M_{\mathrm{H}_{2}}=\alpha_{\mathrm{CO}} L_{\mathrm{CO}}^{\prime}$. However, the ISM mass estimate relies on several assumptions and systematic uncertainties. For instance, to translate observations at different restframe wavelengths into luminosities at $850 \mu \mathrm{m}$, the observed dust emission needs to be modeled, and the common assumption is that of a single-temperature modified blackbody in the long-wavelength optically thin regime. This in turn requires the assumption of a dust absorption coefficient and a dust temperature (e.g., Bianchi 2013). Neither of these parameters is well known, and they can vary for different classes of galaxies. Moreover, the conversion factor $\alpha_{\mathrm{CO}}$ is affected by large uncertainties and likely depends on local ISM conditions, such as pressure, gas dynamics, and metallicity (e.g., Carilli \& Walter 2013, and references therein). Highly star-forming systems usually show values in the range $0.3-1.3$ (Carilli \& Walter 2013), with an average value of $\alpha_{\mathrm{CO}}=0.8 M_{\odot} /\left(\mathrm{K} \mathrm{km} \mathrm{s}^{-1} \mathrm{pc}^{2}\right)$ (e.g., Tacconi et al. 2008; Magdis et al. 2012; Magnelli et al. 2012; Bothwell et al. 2013). This is lower than what is observed in normal galaxies $\left(\alpha_{\mathrm{CO}}=4.5\right)$, implying more $\mathrm{CO}$ emission per unit molecular gas mass, and it is likely related to the different physical conditions in the ISM (e.g., Papadopoulos et al. 2012). We therefore assumed $\alpha_{\mathrm{CO}}=0.8 \pm 0.5 M_{\odot} /\left(\mathrm{K} \mathrm{km} \mathrm{s}^{-1} \mathrm{pc}^{2}\right)$ for our highly starforming systems. Moreover, to account for the atomic hydrogen mass $M_{\mathrm{HI}}$, we considered the results by Calura et al. (2014). They converted the $[\mathrm{CII}] 158 \mu \mathrm{m}$ line luminosity into atomic gas mass for a sample of high- $z$ AGN host galaxies, obtaining an average ratio $M_{\mathrm{H}_{2}} / M_{\mathrm{HI}} \sim 5$ for the whole sample. Theoretical results agree with this estimate (e.g., Lagos et al. 2011). Our gas masses, obtained as the sum of the molecular and neutral hydrogen masses $M_{\mathrm{gas}}=M_{\mathrm{H}_{2}}+M_{\mathrm{HI}}$, are in the range $(0.8-5.4) \times 10^{10} M_{\odot}$, as reported in Table 4. Errors take into account a $20 \%$ error on the luminosity at $850 \mu \mathrm{m}$, the $0.2 \mathrm{dex}$ dispersion of the Scoville et al. (2016) relation, and the range of $\alpha_{\mathrm{CO}}$ values mentioned above.

\section{Discussion}

\subsection{Size of the host galaxies}

In order to infer the column density of the ISM in the host galaxy, we need to estimate the gas extension. As a probe of the gas size, we can consider observations of the thermal FIR continuum, produced by dust heated by young, massive stars, hence representing the regions of active star formation; $\mathrm{CO}$ transitions, tracing molecular gas that serves as the fuel for star formation; and [C II] line emission, probing the photodissociation regions (PDRs) and the interstellar medium.
High-redshift QSOs and SMGs tend to have significant masses of cold dust $\left(M_{\text {dust }} \sim 10^{8}-10^{9} M_{\odot}\right)$ as well as substantial reservoirs of molecular gas $\left(M_{\mathrm{gas}} \sim 10^{10}-10^{11} M_{\odot}\right)$. Several analyses of the stellar component and the molecular gas as well as dust hosted in these sources have confirmed that they have compact sizes. For example, Swinbank et al. (2010) performed a detailed study of the stellar structure for a sample of 25 SMGs (including both AGN and starburst galaxies) at redshift $z \sim 2$. They used deep HST $I$ - and $H$-band images and derived typical half-light radii of about $2 \mathrm{kpc}$. Tacconi et al. (2008) obtained sub-arcsec resolution observations of $\mathrm{CO}$ rotational transitions in four SMGs at $z \sim 2$ using the IRAM Plateau de Bure Interferometer (PdBI). The observed emission had a compact intrinsic size, with $r_{\text {half }} \lesssim 2 \mathrm{kpc}$. Half-light radii in the range $\sim 1-4 \mathrm{kpc}$ have also been measured for the ISM of very distant $(z \sim 6-7)$ quasar hosts by means of ALMA observations of the [C $\mathrm{II}]$ fine structure line and dust continuum emission (see e.g., Decarli et al. 2018; Venemans et al. 2018 and references therein). Other compelling results are provided by Harrison et al. (2016), who presented high-resolution ALMA $870 \mu \mathrm{m}$ imaging of five high-redshift $(z \sim 1.5-4.5)$ AGN host galaxies. They measured angular sizes of $\sim 0.2-0.5^{\prime \prime}$ for the rest-frame FIR emission of their targets, corresponding to star formation scales of $1-3 \mathrm{kpc}$. However, FIR/submm observations at these redshifts are usually just marginally resolved, and therefore these data do not probe the source morphology and just place tight constraints on the spatial extent of the observed objects. All these sources are characterized by physical parameters similar to those derived for our targets, which means stellar masses $M_{*} \sim 10^{10}-10^{11} M_{\odot}$, gas masses $M_{\text {gas }} \sim 10^{10}-10^{11} M_{\odot}$, and IR luminosities $L_{\mathrm{IR}} \gtrsim 10^{12} L_{\odot}$, some of them showing obscured AGN activity detected through X-ray observations.

We lack measurements that would trace the gas component for our sample. We assumed that the molecular gas and dust are cospatial. Although not many observations have so far probed both the dusty and molecular component for targets similar to ours, some works have reported compact sizes of the molecular gas and similar or slightly smaller extensions of the dust (e.g., Hodge et al. 2015; Spilker et al. 2016; Tadaki et al. 2017a,b; Talia et al. 2018, but see also Calistro Rivera et al. 2018). Therefore, we used the size of the dust-emitting region as a probe of the gas size for the sources for which this information is available (see Table 5). For the remaining targets, we assumed that the size of the heated-dust region is half of that of the total stellar emission (e.g., Tadaki et al. 2017b) as derived from CANDELS HST $H$-band data (i.e., rest-frame optical) using GALFIT (van der Wel et al. 2012). This assumption is in agreement with the results of Hodge et al. (2016): by means of ALMA observations at $0.16^{\prime \prime}$ resolution, they found that for the distant (median redshift 2.6) SMGs in the ALESS survey, the size of the central dusty and starbursting region is on average $\sim 2.5$ times smaller than that of stellar emission as measured in CANDELS. These results have been confirmed by Fujimoto et al. (2017) on a larger sample of star-forming galaxies at a similar median redshift and observed by both ALMA and HST. In spite of a large scatter in their measurements, these authors found that FIR-measured sizes are on average $\sim 1.5$ times smaller than those measured at UV/optical (rest-frame) wavelengths.

We have solid multiwavelength observational constraints on the extension of the source XID539 from previous works. De Breuck et al. (2014) presented ALMA Band 7 (345 GHz, i.e., $870 \mu \mathrm{m})$ observations of the [C II] line emission and dust continuum, which are confined in a region with a radius smaller than $2 \mathrm{kpc}$. A continuum Band $6(230 \mathrm{GHz}, 1300 \mu \mathrm{m})$ observation of this target was analyzed by Gilli et al. (2014), who 
Table 5. Half-light radii and column densities derived from SED-fitting and X-ray analyses.

\begin{tabular}{lccccc}
\hline \hline $\begin{array}{l}\mathrm{XID} \\
(1)\end{array}$ & $\begin{array}{c}r_{\text {half," }}^{*} \\
(2)\end{array}$ & $\begin{array}{c}r_{\text {half," }}^{\mathrm{d}}(3) \\
42\end{array}$ & $\begin{array}{c}r_{\text {half,kpc }}^{\mathrm{ISM}} \\
(4)\end{array}$ & $\begin{array}{c}N_{\mathrm{H}, \mathrm{ISM}} \\
(5)\end{array}$ & $\begin{array}{c}N_{\mathrm{H}, \mathrm{X}}{ }^{a} \\
(6)\end{array}$ \\
\hline 170 & $0.16 \pm 0.01$ & $0.15 \pm 0.07$ & $1.2 \pm 0.4$ & $6.0 \pm 5.9$ & $2.0_{-0.9}^{+1.0}$ \\
337 & $0.11 \pm 0.01$ & - & $0.7 \pm 0.3$ & $3.2 \pm 2.7$ & $0.7_{-0.1}^{+0.1}$ \\
539 & $<0.2$ & $0.13 \pm 0.04$ & $0.4 \pm 0.2$ & $8.2 \pm 6.9$ & $10.0_{-2.0}^{+3.0}$ \\
551 & $0.14 \pm 0.01$ & - & $0.5 \pm 0.2$ & $5.2 \pm 8.3$ & $17.0_{-6.8}^{+11.7}$ \\
666 & $0.38 \pm 0.01$ & $0.08 \pm 0.02$ & $0.6 \pm 0.3$ & $10.0 \pm 7.8$ & $32.8_{-8.4}^{+15.4}$ \\
746 & $0.14 \pm 0.01$ & - & $0.5 \pm 0.2$ & $4.2 \pm 3.5$ & $5.5_{-0.5}^{+0.6}$ \\
\hline
\end{tabular}

Notes. (1) X-ray source ID; (2) stellar half-light radius in arcsec as derived from HST/WFC3 $H$-band observations (see Chen et al. $2015 \mathrm{a}$ for XID42 and XID539, and van der Wel et al. 2012 for the remaining sources); (3) dust continuum half-light radius in arcsec from ALMA data (see text); and (4) ISM half-light radius in kpc. We assumed $r_{\text {half }}^{\text {ISM }}=r_{\text {half }}^{\mathrm{d}}$ when ALMA data were available and $r_{\text {half }}^{\text {ISM }}=r_{\text {half }}^{*} / 2$ (with $\sim 30 \%$ errors, similar to those on $r_{\text {half }}^{\mathrm{d}}$ ) otherwise (see text for details); (5) column density associated with the ISM of the host galaxy in units of $10^{23} \mathrm{~cm}^{-2}$; and $(6)$ column density derived from the X-ray spectral analysis in units of $10^{23} \mathrm{~cm}^{-2} .{ }^{(a)}$ Because the geometry adopted for the host ISM is similar to that assumed by the transmission-dominated model used to analyze X-ray spectra, we consider as fiducial results for $N_{\mathrm{H}, \mathrm{X}}$ those derived using the transmission model (see Table 3).

found an intrinsic source size of $0.27 \pm 0.08$ arcsec (Gaussian FWHM), corresponding to a dust half-light radius of $r_{\text {half }}^{\mathrm{d}}=0.9 \pm$ $0.3 \mathrm{kpc}$ (see also Hodge et al. 2016). In the HST/WFC3 $H$-band ( $\sim 2800 \AA$ rest-frame), the source is not resolved, which places an upper limit on the UV rest-frame emission of $1.2 \mathrm{kpc}$ (Chen et al. 2015a). As discussed in Gilli et al. (2014), we assumed a stellar half-light radius of $r_{\text {half }}^{*} \sim 1 \mathrm{kpc}$, which is comparable to what has been found for the dust emission. For XID42 and XID666, we used data from a recent ALMA Band 4 observation at $0.15^{\prime \prime}$ resolution (PI: Gilli). The two sources appear resolved in the ALMA data, with half-light radii for the dust-continuum emission of about $1.2 \pm 0.4$ and $0.6 \pm 0.3 \mathrm{kpc}$, respectively (D'Amato et al., in prep.). These values are lower than the half-light radii of the stellar component in the HST/WFC3 $H$-band, which are $2.2 \pm 0.1 \mathrm{kpc}$ (Chen et al. 2015a) and $\sim 3 \mathrm{kpc}$ (van der Wel et al. 2012) for XID42 and XID666, respectively. Overall, for the three sources in our sample with both high-resolution ALMA and HST $H$-band data, the rest-FIR size is from 1.1 to 5 times smaller than the rest-optical size, in agreement with the general trend found in the literature, and again indicating that SMGs have central dusty starbursts that are more compact than the whole stellar distribution. For the remaining sources, XID170, XID337, XID551, and XID746, we assumed that the extension of the ISM is half of that measured for the total stellar emission, as derived from CANDELS HST $H$-band data (van der Wel et al. 2012). The adopted ISM half-light radii are reported in Table 5.

\subsection{ISM column density}

In order to estimate the equivalent column density associated with the gas in the host galaxy, we considered a simple geometrical approximation assuming a spherical gas distribution with uniform density. Hence, under the assumption that both molecular and atomic gas are cospatial with dust, and considering that half of the total gas mass $M_{\mathrm{gas}}=M_{\mathrm{H}_{2}}+M_{\mathrm{HI}}$ is confined within $r_{\text {half }}^{\mathrm{ISM}}$, we computed the ISM column densities for the seven sources of our sample:

$N_{\mathrm{H}}=\int_{0}^{r_{\mathrm{h}}} n_{\mathrm{H}} \mathrm{d} s$

which is the volume density $n_{\mathrm{H}}$ of hydrogen atoms inside the sphere of radius $r_{\mathrm{h}}$ integrated over the path length ds.
The values obtained are reported in Table 5 and range between $\sim 10^{23}$ and $\sim 10^{24} \mathrm{~cm}^{-2}$, which are on the same order as those derived from the X-ray spectral analysis. Because the geometry adopted for the host ISM is similar to that assumed by the transmission-dominated model used to analyze the X-ray spectra, we consider as fiducial results for $N_{\mathrm{H}, \mathrm{X}}$ those derived by using the transmission model (see Table 3). The outcome of this comparison is that the host ISM can significantly contribute to the observed X-ray obscuration.

In Fig. 3 the ISM column densities are plotted against the values derived from the X-ray spectral analysis. For the four sources without any direct measurement of the FIR-rest size, we also plot (open circles) the ISM column density that would be obtained by assuming a dust-to-stellar size ratio of 1 instead of 0.5 . Clearly, the derived ISM columns would decrease by a factor of 4. Moreover, we note that Calistro Rivera et al. (2018) reported a $\mathrm{CO}(3-2)$ half-light radius for the target XID42 of $3.1 \pm 0.5 \mathrm{kpc}$. By assuming this value, the column density would decrease by a factor of 7 , but still represent $40 \%$ of the value derived from the X-rays. In general, the similarity between the ISM column densities and the X-ray column densities suggests that the host ISM is capable of providing significant absorption on large (kpc) scales that adds to (or even replaces) the absorption produced on small (pc) scales by any circumnuclear material (i.e., the torus). The presence of hot dust surrounding the central engine and heated by its emission is indeed supported by the mid-IR "excess" observed in the SED. This component, which is also observed in X-ray unobscured AGN, does not necessarily account for the whole obscuration.

If we consider that complex merging phenomena, gas inflows toward the central engine and inhomogeneous collapse of gas are expected during the evolution of these sources (e.g., Hopkins et al. 2006; Lapi et al. 2014), a simple unification model based on torus-like absorbers and ordered gas motions may not apply, and nuclear radiation may then be absorbed by gas located at different physical scales. In particular, the medium in the host galaxy is an ingredient that should be considered, as also suggested by the evidence for an increase in X-ray column density with the stellar mass of the AGN host, as has recently been found by Buchner \& Bauer (2017) and Lanzuisi et al. (2017).

Hopkins et al. $(2005,2006)$ studied the AGN obscuration during a major merger event by computing the column density 


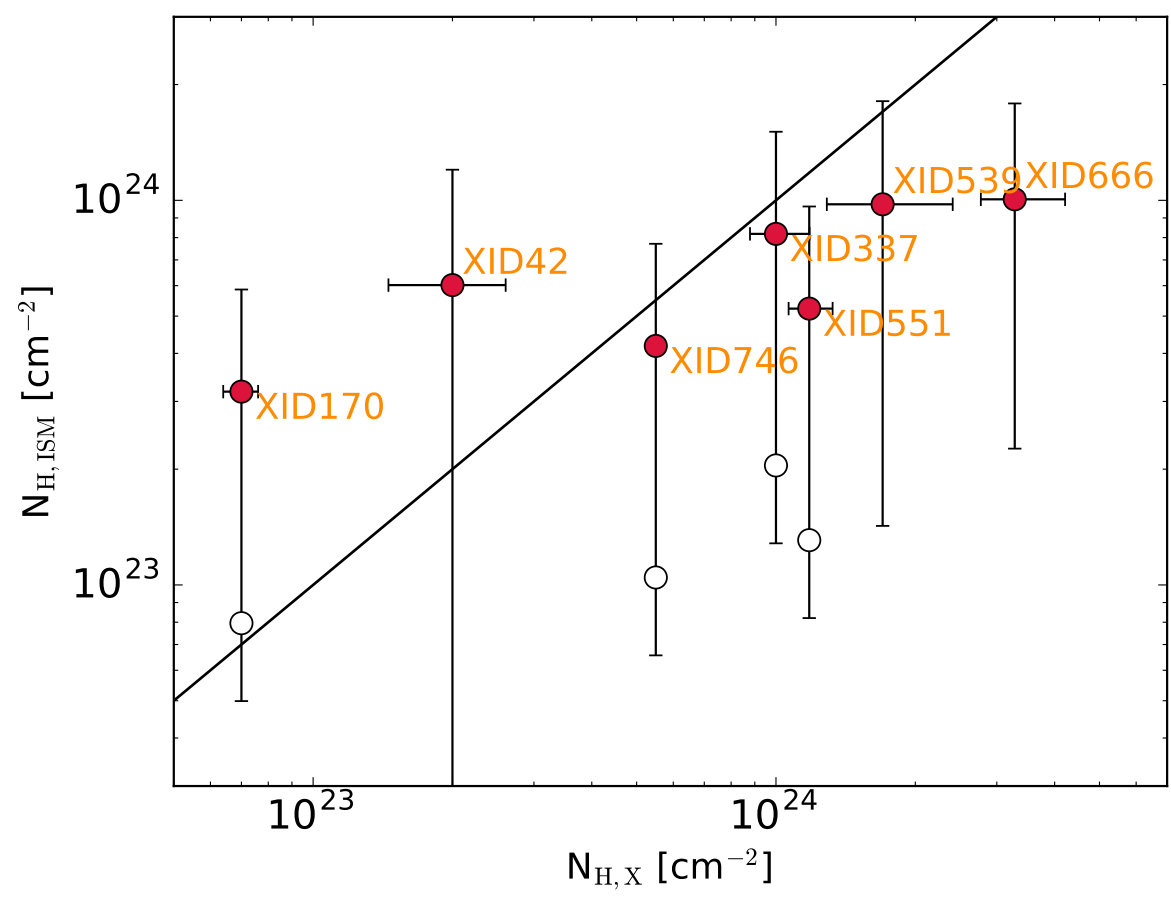

Fig. 3. ISM column density vs. X-ray column density for our targets. The solid line shows the 1:1 relation. Filled circles were derived assuming $r_{\text {half }}^{\mathrm{ISM}}$, as reported in Table 5. For the targets with no ALMA data, the ISM densities that would be obtained by assuming $r_{\text {half }}^{\text {ISM }}=r_{\text {half }}^{*}$ instead of $r_{\text {half }}^{\mathrm{ISM}}=r_{\text {half }}^{*} / 2$ are also shown as open circles. along several lines of sight and modeling the ISM through a hot (diffuse) and a cold (molecular and neutral) phase, with most of the mass distributed in dense and cold structures. They found that the column density does not depend considerably on the assumptions regarding the small-scale physics of the ISM and obscuration, as the central regions of the merging galaxies are expected to be highly chaotic. Moreover, the scales associated with obscuration are related to starburst activity and obscured quasar growth, and were found to be larger $(z 100 \mathrm{pc})$ than the typical scales of traditional tori. In particular, the galaxy ISM is able to generate an important contribution to the obscuration across most lines of sight toward the nucleus (Trebitsch et al. 2019) (a few sightlines may still be free from obscuration as witnessed in, e.g., unobscured quasars hosted by gas-rich galaxies, Fu et al. 2017; Decarli et al. 2018). In this scenario, the obscuring column density could be an evolving function of time, luminosity, and host galaxy properties, dominated by gas inflows that fuel the central BH in different evolutionary stages. In particular, because of the larger gas content and smaller size of highredshift galaxies, kpc-scale obscuration by the host ISM may be responsible for the observed increase of the obscured AGN fraction toward high redshifts (Vito et al. 2014, 2018; Aird et al. 2015; Buchner et al. 2015).

The role of the host galaxy ISM in obscuring the AGN emission has also been studied through numerical simulations (e.g., Bournaud et al. 2011a,b). Thick gaseous disks in high-redshift galaxies subject to violent instability can produce strong obscuration toward the central AGN, characterized by very high column densities $\left(N_{\mathrm{H}}>10^{23} \mathrm{~cm}^{-2}\right)$ that even reach the Compton-thick regime. Juneau et al. (2013) pointed out that at high redshift, in addition to small-scale absorption (i.e., the pc-scale torus), large amounts of gas in galaxies might contribute to absorbing X-rays. In particular, they found a more frequent X-ray absorption in galaxies hosting an AGN with higher sSFRs (i.e., SFR/ $M_{*}$ ). A possible explanation for this observed trend is that the gas reservoir that fuels the intense star formation also acts as a relevant source of obscuration for the AGN. This situation could be more likely at high redshift, where the AGN hosts show an increase in SFR and gas content (e.g., Carilli \& Walter 2013).
The kinematics and spatial distribution of the ISM might be better constrained by observing molecular lines, which provide the most direct insight into the physics and behavior of these systems. SMGs and QSOs often exhibit double-peaked $\mathrm{CO}$ spectra, which are a potential indicator of either the existence of kinematically distinct components within these systems or a rotating disk-like component (e.g., Tacconi et al. 2008; Bothwell et al. 2013). However, galaxy-integrated line fluxes are mainly measured at high redshift, and spatially resolved molecular gas observations are restricted to a few bright sources (Carilli \& Walter 2013). Therefore, inferring the size of the CO reservoir and studying the kinematic mode that determines the gas dynamics, as well as how the ISM takes part in the obscuration of the central AGN, is very challenging at these redshifts.

\subsection{Possible progenitors of the cQGs}

We constrained the surface densities of SFRs, gas, and stellar masses as derived from SED fitting (see Table 4), assuming a uniform distribution with radius $r_{\text {half }}$. The results are reported in Table 6 . The surface density of star formation, $\Sigma_{\mathrm{SFR}}=$ $(\mathrm{SFR} / 2) /\left(\pi r_{\text {half }}^{2}\right)$, ranges between $\sim 108$ and $\sim 338 M_{\odot} \mathrm{yr}^{-1} \mathrm{kpc}^{-2}$, in line with the range found by, for example, Harrison et al. (2016) for a sample of X-ray selected AGN at $z \sim 1.5-4.5$ and observed with ALMA (see also Genzel et al. 2010; Hodge et al. 2013). Similarly, we estimated the gas surface density, $\Sigma_{\text {gas }}=\Sigma_{\mathrm{HI}+\mathrm{H}_{2}}=\left(M_{\text {gas }} / 2\right) /\left(\pi r_{\text {half }}^{2}\right)$, with values in the range $(0.3-1.1) \times 10^{10} M_{\odot} \mathrm{kpc}^{-2}$. These values are in agreement with those typically found for SMGs (see, e.g., Daddi et al. 2010 and Swinbank et al. 2010 for a comparison with $\Sigma_{\text {gas }}$ and $\Sigma_{*}$ obtained for SMGs). Finally, we combined the size and stellar mass estimates to derive the stellar surface density, $\Sigma_{*}=\left(M_{*} / 2\right) /\left(\pi r_{\text {half }}^{2}\right)$, where $r_{\text {half }}$ is the stellar half-light radius. Our results are in the range $(0.7-8.7) \times 10^{10} M_{\odot} \mathrm{kpc}^{-2}$ : the stellar density is about one dex higher than what is found for local elliptical galaxies of similar mass (e.g., as derived by Hopkins et al. 2010 based on the HST data of Lauer et al. 2007), and is instead similar to what is found in compact quiescent galaxies (cQGs) at $z \gtrsim 1$ (Trujillo et al. 2007; Cimatti et al. 2008). 
Table 6. Surface densities of SFR, gas, and stars together with gas depletion timescales of the target sample.

\begin{tabular}{lcccc}
\hline $\begin{array}{l}\text { XID } \\
(1)\end{array}$ & $\begin{array}{c}\Sigma_{\text {SFR }} \\
(2)\end{array}$ & $\begin{array}{c}\Sigma_{\text {gas }} \\
(3)\end{array}$ & $\begin{array}{c}\Sigma_{*} \\
(4)\end{array}$ & $\begin{array}{c}t_{\text {dep }} \\
(5)\end{array}$ \\
\hline 42 & $198 \pm 41$ & $6.4 \pm 3.8$ & $0.7 \pm 0.2$ & $3.2 \pm 2.0$ \\
170 & $108 \pm 23$ & $3.4 \pm 2.0$ & $1.7 \pm 0.5$ & $3.1 \pm 2.0$ \\
337 & $195 \pm 40$ & $8.7 \pm 5.1$ & $8.7 \pm 2.6$ & $4.5 \pm 2.8$ \\
539 & $169 \pm 41$ & $10.4 \pm 6.1$ & $2.1 \pm 0.6$ & $5.3 \pm 3.3$ \\
551 & $288 \pm 59$ & $5.5 \pm 3.3$ & $3.5 \pm 1.0$ & $1.9 \pm 1.2$ \\
666 & $338 \pm 69$ & $10.7 \pm 6.3$ & $0.8 \pm 0.2$ & $3.2 \pm 2.0$ \\
746 & $166 \pm 35$ & $4.4 \pm 2.6$ & $6.0 \pm 1.8$ & $2.7 \pm 1.7$ \\
\hline
\end{tabular}

Notes. (1) X-ray source ID; (2) SFR surface density in units of $M_{\odot} \mathrm{yr}^{-1} \mathrm{kpc}^{-2}$; (3) gas surface density in units of $10^{9} M_{\odot} \mathrm{kpc}^{-2}$; (4) stellar surface density in units of $10^{10} M_{\odot} \mathrm{kpc}^{-2}$; and (5) depletion timescale in units of $10^{7} \mathrm{yr}$.

High-redshift QSOs and SMGs are thought to be complex systems of dense gas, massive star formation, and even AGN activity. Tacconi et al. (2008) suggested that a significant fraction of the stellar mass of these objects $(\sim 50 \%)$ formed and assembled during their most active phase, while the rest formed over a longer period of time. Taking into account the high SFRs, we can estimate the gas depletion timescale, that is, the time that the available gas needs to be depleted assuming a constant SFR. Hence, $t_{\mathrm{dep}}=M_{\mathrm{gas}} / \mathrm{SFR}$ is found to be in the range $(2-5) \times 10^{7}$ yr. After the SMG phase, which ends when the gas supply is depleted or if star formation is quenched and further prevented by negative feedback from AGN and supernovae, the galaxy will end up as a compact passive system (e.g., Lapi et al. 2014). It has been argued that SMGs at high redshift (e.g., Tacconi et al. 2008; Gilli et al. 2014) could be the best progenitors of cQGs (see, e.g., Barro et al. 2013; Fu et al. 2013) observed at $1 \lesssim z \lesssim 3$. These objects indeed show compact morphologies, with stellar half-light radii $r_{\text {half }} \sim 0.5-2 \mathrm{kpc}$ and stellar surface densities $\Sigma_{*}>10^{10} M_{\odot} \mathrm{kpc}^{-2}$. The formation channels of the cQGs are still an open issue. However, we showed that our sources have a compact (sub-kpc for most of the sample) stellar core with stellar surface densities similar to those of cQGs observed at $z>1$. This is in line with what has been found by Toft et al. (2014), who compared the properties of a sample of $z \gtrsim 3$ SMGs and $z \sim 2$ cQGs and concluded that SMGs evolve into cQGs. Based on the comoving number density of their samples, Toft et al. (2014) derived an SMG duty cycle of $\sim 42 \mathrm{Myr}$, which is in agreement with our estimates of the gas depletion timescale but is still independent of the arguments we used. According to the values derived for SFR, stellar mass, gas depletion timescale, and size, our targets could therefore be the progenitors of this type of systems.

\section{Conclusions}

We have presented a multiwavelength analysis of a sample of seven heavily obscured AGN and their host galaxies at high redshift in the CDF-S, which were selected because they have good detections in the FIR domain. By exploiting the superb datasets that are available in this field (spanning from the ultradeep $7 \mathrm{Ms}$ Chandra exposure to the broadband photometry of HST/CANDELS and Herschel, as well as ALMA), we were able to characterize the physical properties of the active nuclei and their hosts, and to place constraints on the role of the host ISM in obscuring the AGN. Our results are summarized below.
- We extracted the X-ray spectra from the $7 \mathrm{Ms}$ Chandra dataset and derived obscuring column densities in the range $N_{\mathrm{H}}=(0.07-3) \times 10^{24} \mathrm{~cm}^{-2}$ and intrinsic rest-frame luminosities in the range $L_{[2-10 \mathrm{keV}]}=(2-7) \times 10^{44} \mathrm{erg} \mathrm{s}^{-1}$. Moreover, we found that most of our targets feature prominent iron $\mathrm{K} \alpha$ lines with $\mathrm{EW} \gtrsim 0.5 \mathrm{keV}$, as expected in heavily obscured nuclei. Our combined X-ray and FIR selection hence returned a sample made only of obscured AGN, which indicates a connection between the dust and gas content in the host ISM and nuclear obscuration.

- We built up the UV-to-FIR SEDs for our targets and analyzed them by means of an SED decomposition technique, from which we derived stellar masses in the range $M_{*}=$ $(1.7-4.4) \times 10^{11} M_{\odot}$, total IR $(8-1000 \mu \mathrm{m})$ luminosities $L_{\mathrm{IR}}=(1.0-9.6) \times 10^{12} L_{\odot}$, and AGN bolometric luminosities $L_{\text {bol }}=(1.9-4.8) \times 10^{12} L_{\odot}$. Moreover, by subtracting the AGN contribution to the total IR luminosity, we measured star formation rates in the range SFR $=190-1680 M_{\odot} \mathrm{yr}^{-1}$.

- We estimated the gas content of the host galaxies using the Scoville et al. (2016) calibration, which relates the intrinsic luminosity at $850 \mu \mathrm{m}$ rest frame (derived from the model SED and interpreted as emission from dust heated by star formation) to the molecular gas mass, that is, the fuel for star formation activity. Our targets host large reservoirs of cold gas, with masses $M_{\text {gas }}=(0.8-5.4) \times 10^{10} M_{\odot}$. Under the assumption that the heated dust and gas are confined within regions of comparable size, we used ALMA dust-continuum data to assess this size for three of the seven targets. For the remaining targets, we assumed that the characteristic size of the region containing both gas and dust is about half the size measured by HST/CANDELS for the optical stellar emission, as seen on average in distant SMGs. The estimated ISM half-light radii for our sample are small, ranging between $\sim 0.4$ and $1.2 \mathrm{kpc}$.

- By adopting a simple geometrical model, specifically, a spherical gas distribution of uniform gas, we computed the column densities associated with the ISM of the host galaxy and showed that they are comparable to those measured from the X-ray spectral analysis.

Our result suggests that in high-redshift gas-rich systems, the obscuration of the nucleus may occur on large $(\mathrm{kpc})$ scales and be produced by the ISM of the host. Obscuration by the ISM may then add to that produced by a small-scale circumnuclear medium (e.g., the torus of the unified model) and constitutes an important ingredient for understanding the coevolution of galaxies with their black holes.

Acknowledgements. We thank the anonymous referee for carefully reading the paper and providing comments. CC thanks J. Buchner, C.-C. Chen (T.C.), H. Fu, C. M. Harrison, G. Lanzuisi, A. Puglisi, and M. Salvato for helpful discussions, and Q. D'Amato for providing the sizes of XID42 and XID666. CC acknowledges support from the IMPRS on Astrophysics at the LMU (Munich). We acknowledge financial contribution from the agreement ASI-INAF n.201714-H.O and ASI-INAF I/037/12/0. AF acknowledges support from the ERC via Advanced Grant under grants agreement no. 321323-NEOGAL and no. 339659MUSICOS. FC acknowledges funding from the INAF PRIN-SKA 2017 program 1.05.01.88.04. FV acknowledges support from CONICYT and CASSACA through the fourth call for tenders of the CAS-CONICYT Fund. The scientific results reported in this article are based to a significant degree on observations made by the Chandra X-ray Observatory.

\section{References}

Aird, J., Nandra, K., Laird, E. S., et al. 2010, MNRAS, 401, 2531 Aird, J., Coil, A. L., Georgakakis, A., et al. 2015, MNRAS, 451, 1892 
Akylas, A., Georgakakis, A., Georgantopoulos, I., Brightman, M., \& Nandra, K. 2012, A\&A, 546, A98

Allen, R. J., Kacprzak, G. G., Glazebrook, K., et al. 2017, ApJ, 834, L11 Arnaud, K. A. 1996, ASP Conf. Ser., 101, 17

Avni, Y. 1976, ApJ, 210, 642

Baloković, M., Comastri, A., Harrison, F. A., et al. 2014, ApJ, 794, 111

Barro, G., Faber, S. M., Pérez-González, P. G., et al. 2013, ApJ, 765, 104

Bianchi, S. 2013, A\&A, 552, A89

Blain, A. W., Smail, I., Ivison, R. J., Kneib, J. P., \& Frayer, D. T. 2002, Phys Rep., 369, 111

Bothwell, M. S., Smail, I., Chapman, S. C., et al. 2013, MNRAS, 429, 3047

Bournaud, F., Chapon, D., Teyssier, R., et al. 2011a, ApJ, 730, 4

Bournaud, F., Dekel, A., Teyssier, R., et al. 2011b, ApJ, 741, L33

Buchner, J., \& Bauer, F. E. 2017, MNRAS, 465, 4348

Buchner, J., Georgakakis, A., Nandra, K., et al. 2015, ApJ, 802, 89

Burtscher, L., Meisenheimer, K., Tristram, K. R. W., et al. 2013, A\&A, 558, A149

Calistro Rivera, G., Hodge, J. A., Smail, I., et al. 2018, ApJ, 863, 56

Calura, F., Gilli, R., Vignali, C., et al. 2014, MNRAS, 438, 2765

Calzetti, D., Armus, L., Bohlin, R. C., et al. 2000, ApJ, 533, 682

Carilli, C. L., \& Walter, F. 2013, ARA\&A, 51, 105

Cash, W. 1979, ApJ, 228, 939

Chen, C.-C., Smail, I., Swinbank, A. M., et al. 2015a, ApJ, 799, 194

Chen, C.-T. J., Hickox, R. C., Alberts, S., et al. 2015b, ApJ, 802, 50

Cimatti, A., Cassata, P., Pozzetti, L., et al. 2008, A\&A, 482, 21

Comastri, A., Iwasawa, K., Gilli, R., et al. 2010, ApJ, 717, 787

Comastri, A., Ranalli, P., Iwasawa, K., et al. 2011, A\&A, 526, L9

Coppin, K. E. K., Chapman, S. C., Smail, I., et al. 2010, MNRAS, 407, L103

Daddi, E., Elbaz, D., Walter, F., et al. 2010, ApJ, 714, L118

De Breuck, C., Williams, R. J., Swinbank, M., et al. 2014, A\&A, 565, A59

Decarli, R., Walter, F., Venemans, B. P., et al. 2018, ApJ, 854, 97

Del Moro, A., Alexander, D. M., Bauer, F. E., et al. 2016, MNRAS, 456, 2105

Delvecchio, I., Gruppioni, C., Pozzi, F., et al. 2014, MNRAS, 439, 2736

Feltre, A., Hatziminaoglou, E., Fritz, J., \& Franceschini, A. 2012, MNRAS, 426 120

Feltre, A., Hatziminaoglou, E., Hernán-Caballero, A., et al. 2013, MNRAS, 434, 2426

Ferrarese, L., \& Merritt, D. 2000, ApJ, 539, L9

Feruglio, C., Daddi, E., Fiore, F., et al. 2011, ApJ, 729, L4

Fritz, J., Franceschini, A., \& Hatziminaoglou, E. 2006, MNRAS, 366, 767

Fu, H., Cooray, A., Feruglio, C., et al. 2013, Nature, 498, 338

Fu, H., Isbell, J., Casey, C. M., et al. 2017, ApJ, 844, 123

Fujimoto, S., Ouchi, M., Shibuya, T., \& Nagai, H. 2017, ApJ, 850, 83

Gandhi, P., Horst, H., Smette, A., et al. 2009, A\&A, 502, 457

Genzel, R., Tacconi, L. J., Gracia-Carpio, J., et al. 2010, MNRAS, 407, 2091

Giavalisco, M., Ferguson, H. C., Koekemoer, A. M., et al. 2004, ApJ, 600, L93

Gilli, R., Comastri, A., \& Hasinger, G. 2007, A\&A, 463, 79

Gilli, R., Su, J., Norman, C., et al. 2011, ApJ, 730, L28

Gilli, R., Norman, C., Vignali, C., et al. 2014, A\&A, 562, A67

Grogin, N. A., Kocevski, D. D., Faber, S. M., et al. 2011, ApJS, 197, 35

Harrison, C. M., Simpson, J. M., Stanley, F., et al. 2016, MNRAS, 457, L122

Hatziminaoglou, E., Fritz, J., Franceschini, A., et al. 2008, MNRAS, 386 1252

Hatziminaoglou, E., Omont, A., Stevens, J. A., et al. 2010, A\&A, 518, L33

Häussler, B., McIntosh, D. H., Barden, M., et al. 2007, ApJS, 172, 615

Hodge, J. A., Karim, A., Smail, I., et al. 2013, ApJ, 768, 91

Hodge, J. A., Riechers, D., Decarli, R., et al. 2015, ApJ, 798, L18

Hodge, J. A., Swinbank, A. M., Simpson, J. M., et al. 2016, ApJ, 833, 103

Hopkins, P. F., Hernquist, L., Cox, T. J., et al. 2005, ApJ, 630, 705

Hopkins, P. F., Hernquist, L., Cox, T. J., et al. 2006, ApJS, 163, 1

Hopkins, P. F., Bundy, K., Hernquist, L., Wuyts, S., \& Cox, T. J. 2010, MNRAS, 401, 1099

Hsu, L.-T., Salvato, M., Nandra, K., et al. 2014, ApJ, 796, 60

Jaffe, W., Meisenheimer, K., Röttgering, H. J. A., et al. 2004, Nature, 429, 47

Juneau, S., Dickinson, M., Bournaud, F., et al. 2013, ApJ, 764, 176

Kennicutt, Jr., R. C. 1998, ApJ, 498, 541

Kormendy, J., \& Ho, L. C. 2013, ARA\&A, 51, 511

Lagos, C. D. P., Baugh, C. M., Lacey, C. G., et al. 2011, MNRAS, 418, 1649

Lampton, M., Margon, B., \& Bowyer, S. 1976, ApJ, 208, 177

Lanzuisi, G., Delvecchio, I., Berta, S., et al. 2017, A\&A, 602, A123
Lapi, A., Raimundo, S., Aversa, R., et al. 2014, ApJ, 782, 69

Lapi, A., Pantoni, L., Zanisi, L., et al. 2018, ApJ, 857, 22

Lauer, T. R., Gebhardt, K., Faber, S. M., et al. 2007, ApJ, 664, 226

Lehmer, B. D., Brandt, W. N., Alexander, D. M., et al. 2005, ApJS, 161, 21

Liu, T., Tozzi, P., Wang, J.-X., et al. 2017, ApJS, 232, 8

Luo, B., Brandt, W. N., Xue, Y. Q., et al. 2017, ApJS, 228, 2

Lusso, E., Comastri, A., Simmons, B. D., et al. 2012, MNRAS, 425, 623

Madau, P., \& Dickinson, M. 2014, ARA\&A, 52, 415

Magdis, G. E., Daddi, E., Béthermin, M., et al. 2012, ApJ, 760, 6

Magdziarz, P., \& Zdziarski, A. A. 1995, MNRAS, 273, 837

Magnelli, B., Saintonge, A., Lutz, D., et al. 2012, A\&A, 548, A22

Magnelli, B., Popesso, P., Berta, S., et al. 2013, A\&A, 553, A132

Magorrian, J., Tremaine, S., Richstone, D., et al. 1998, AJ, 115, 2285

Mainieri, V., Bergeron, J., Hasinger, G., et al. 2002, A\&A, 393, 425

Mainieri, V., Rigopoulou, D., Lehmann, I., et al. 2005, MNRAS, 356, 1571

Maiolino, R., Salvati, M., Bassani, L., et al. 1998, A\&A, 338, 781

Mateos, S., Barcons, X., Carrera, F. J., et al. 2005, A\&A, 444, 79

Murphy, K. D., \& Yaqoob, T. 2009, MNRAS, 397, 1549

Nandra, K., \& Pounds, K. A. 1994, MNRAS, 268, 405

Nenkova, M., Sirocky, M. M., Ivezić, Ž., \& Elitzur, M. 2008a, ApJ, 685, 147

Nenkova, M., Sirocky, M. M., Nikutta, R., Ivezić, Ž., \& Elitzur, M. 2008b, ApJ, 685,160

Norman, C., Hasinger, G., Giacconi, R., et al. 2002, ApJ, 571, 218

Oliver, S. J., Bock, J., Altieri, B., et al. 2012, MNRAS, 424, 1614

Papadopoulos, P. P., van der Werf, P. P., Xilouris, E. M., et al. 2012, MNRAS, 426, 2601

Planck Collaboration XIII. (Ade, P. A. R., et al.) 2016, A\&A, 594, A13

Polletta, M., Tajer, M., Maraschi, L., et al. 2007, ApJ, 663, 81

Pozzi, F., Vignali, C., Gruppioni, C., et al. 2012, MNRAS, 423, 1909

Privon, G. C., Narayanan, D., \& Davé, R. 2018, ApJ, 867, 102

Ricci, C., Trakhtenbrot, B., Koss, M. J., et al. 2017, ApJS, 233, 17

Rigopoulou, D., Mainieri, V., Almaini, O., et al. 2009, MNRAS, 400, 1199

Risaliti, G., \& Risaliti, G. Astrophys. Space Sci. Lib., 308, 187

Salpeter, E. E. 1955, ApJ, 121, 161

Santini, P., Ferguson, H. C., Fontana, A., et al. 2015, ApJ, 801, 97

Schlegel, D. J., Finkbeiner, D. P., \& Davis, M. 1998, ApJ, 500, 525

Scoville, N., Sheth, K., Aussel, H., et al. 2016, ApJ, 820, 83

Scoville, N., Lee, N., Vanden Bout, P., et al. 2017, ApJ, 837, 150

Shibuya, T., Ouchi, M., \& Harikane, Y. 2015, ApJS, 219, 15

Simpson, J. M., Swinbank, A. M., Smail, I., et al. 2014, ApJ, 788, 125

Spilker, J. S., Bezanson, R., Marrone, D. P., et al. 2016, ApJ, 832, 19

Stark, A. A., Gammie, C. F., Wilson, R. W., et al. 1992, ApJS, 79, 77

Swinbank, A. M., Smail, I., Chapman, S. C., et al. 2010, MNRAS, 405, 234

Szokoly, G. P., Bergeron, J., Hasinger, G., et al. 2004, ApJS, 155, 271

Tacconi, L. J., Genzel, R., Smail, I., et al. 2008, ApJ, 680, 246

Tacconi, L. J., Genzel, R., Saintonge, A., et al. 2018, ApJ, 853, 179

Tadaki, K.-I., Genzel, R., Kodama, T., et al. 2017a, ApJ, 834, 135

Tadaki, K.-I., Kodama, T., Nelson, E. J., et al. 2017b, ApJ, 841, L25

Talia, M., Pozzi, F., Vallini, L., et al. 2018, MNRAS, 476, 3956

Toft, S., Smolčić, V., Magnelli, B., et al. 2014, ApJ, 782, 68

Tozzi, P., Gilli, R., Mainieri, V., et al. 2006, A\&A, 451, 457

Trebitsch, M., Volonteri, M., Dubois, Y. 2019, MNRAS, submitted [arXiv:1901.01261]

Tristram, K. R. W., Meisenheimer, K., Jaffe, W., et al. 2007, A\&A, 474, 837

Trujillo, I., Conselice, C. J., Bundy, K., et al. 2007, MNRAS, 382, 109

Ueda, Y., Akiyama, M., Hasinger, G., Miyaji, T., \& Watson, M. G. 2014, ApJ, 786, 104

Urry, C. M., \& Padovani, P. 1995, PASP, 107, 803

van der Wel, A., Bell, E. F., Häussler, B., et al. 2012, ApJS, 203, 24

van der Wel, A., Franx, M., van Dokkum, P. G., et al. 2014, ApJ, 788, 28

Venemans, B., Decarli, R., Walter, F., et al. 2018, ApJ, 866, 159

Vignali, C., Piconcelli, E., Lanzuisi, G., et al. 2011, MNRAS, 416, 2068

Vito, F., Vignali, C., Gilli, R., et al. 2013, MNRAS, 428, 354

Vito, F., Gilli, R., Vignali, C., et al. 2014, MNRAS, 445, 3557

Vito, F., Brandt, W. N., Yang, G., et al. 2018, MNRAS, 473, 2378

Wachter, K., Leach, R., \& Kellogg, E. 1979, ApJ, 230, 274

Wang, R., Wagg, J., Carilli, C. L., et al. 2013a, ApJ, 773, 44

Wang, S. X., Brandt, W. N., Luo, B., et al. 2013b, ApJ, 778, 179

Xue, Y. Q., Luo, B., Brandt, W. N., et al. 2011, ApJS, 195, 10

Xue, Y. Q., Luo, B., Brandt, W. N., et al. 2016, ApJS, 224, 15

Yaqoob, T. 1997, ApJ, 479, 184 


\section{Appendix A: Notes on individual targets}

\section{A.1. XID42}

This target has the largest off-axis angle of the sample in the CDF-S, therefore it is strongly background dominated, and the useful energy range for a spectral analysis is reduced to $0.8-3 \mathrm{keV}$. A second power law emerging in the soft X-rays was not required by the fit. We note a difference by a factor $\sim 2$ in the observed flux derived using the reflection model with respect to the transmission and MYTorus models. This might be ascribed to the different shape of this model compared to the others outside the covered energy range. The $\mathrm{Fe} \mathrm{K} \alpha$ line is detected at a rest-frame energy of about $6.5 \mathrm{keV}$ at $\sim 2.2 \sigma$; its EW is characterized by large errors that are due to the poor spectral quality.

\section{A.2. XID170}

The X-ray data of XID170 are well fit by the transmission and MYTorus models, but not by the reflection model, whose best-fit parameters significantly differ from those derived with the other two models. A second power law was not required to improve the fit quality in the soft $\mathrm{X}$-ray regime. The iron line is poorly constrained.

\section{A.3. $X I D 337$}

The best-fit parameters obtained by analyzing the X-ray spectrum with the whole set of models agree well. The $\mathrm{Fe} \mathrm{K} \alpha$ line parameters are constrained in the transmission and MYTorus models, while its energy was fixed to the best-fit value in the reflection model; in this case, only an upper limit on its EW is computed. We added a soft component to the transmission and MYTorus models, and the resulting fraction of scattered emission, computed as the ratio between the power-law normalizations in the MYTorus model, is $\sim 3 \%$. The derived best-fit column densities point to a Compton-thick emission.

The SED of this target represents a particular case in our sample. Thanks to the high resolution $\left(\sim 0.2^{\prime \prime}\right)$ of the ALMA image at $870 \mu \mathrm{m}$, we have found that this source, which was thought to be a single object in the Spitzer/MIPS and Herschel maps (and in SCUBA observations, Mainieri et al. 2005), is actually a blend of two sources. The MIR and FIR photometry is dominated by a bright source at $3.5^{\prime \prime}$ from the target and forced us to convert these data points into upper limits. As a result, this translates into a very uncertain AGN contribution.

\section{A.4. XID539}

This source lies at a very large off-axis position with respect to the center of the CDF-S and is characterized by low photon statistics. The iron emission line is detected at $\sim 6.9 \mathrm{keV}$ (at $\sim 2 \sigma$ ) with the transmission model, while is kept fixed to the best-fit value in the reflection model. As for MYTorus, we added a Gaussian component to reproduce the line at such energies because the default line energy is $6.4 \mathrm{keV}$ (i.e., due to neutral iron). The energy of the line can be interpreted as emission from highly ionized iron (i.e., hydrogen-like iron) and is also very prominent according to the derived EW.

\section{A.5. XID551}

This target lies in the inner region of the CDF-S area and has reasonably good photon statistics. The secondary power law accounts for about $3 \%$ of the unobscured flux at $1 \mathrm{keV}$. The emission line, detected at $\sim 3 \sigma$ and at $\sim 6.6 \mathrm{keV}$ rest-frame, could be ascribed to emission from either neutral or ionized iron (i.e., helium-like iron) or a mixture of the two.

\section{A.6. XID666}

XID666 is the most obscured source of the sample. The high column density results in a lower limit in MYTorus because of the low photon statistics. The resulting fraction of scattered emission in the soft X-rays is smaller than $1 \%$. The target is characterized by a flat spectrum and an extremely strong iron $\mathrm{K} \alpha$ line at $\sim 6.4 \mathrm{keV}$, clearly detected at $\sim 4.5 \sigma$. The EW is well constrained and larger than $1 \mathrm{keV}$. All models are in agreement in terms of flux and luminosities. This is suggestive of a spectrum dominated by reflected emission.

\section{A.7. $X I D 746$}

Like XID170, the reflection model of XID746 does not provide a good fit to the spectral data. The transmission model provides a best-fit column density of $\sim 5.5 \times 10^{23} \mathrm{~cm}^{-2}$. The iron line is not required by the data, therefore only an upper limit on the EW is reported. 